\title{
Zero temperature quantum properties of small protonated water clusters $\left(\mathrm{H}_{2} \mathrm{O}\right){ }_{n} \mathrm{H}^{+}(n=1-5)$
}

\author{
Massimo Mella ${ }^{\text {a) }}$ and David C. Clary \\ Physical and Theoretical Chemistry Laboratory, Department of Chemistry, University of Oxford, \\ South Parks Road, Oxford OX1 3QZ, United Kingdom
}

(Received 22 May 2003; accepted 22 August 2003)

\begin{abstract}
The study of the energetics and structure of small protonated water clusters $\left(\mathrm{H}_{2} \mathrm{O}\right)_{n} \mathrm{H}^{+}(n$ $=1-5$ ) has been carried out employing the OSS3 potential energy surface developed by Ojamae, Singer, and Shavitt [J. Chem. Phys. 109, 5547 (1998)]. By comparing it with accurate ab initio MP2 calculations for $\left(\mathrm{H}_{2} \mathrm{O}\right)_{n} \mathrm{H}^{+}$, this all-atom potential is also shown to reproduce quantitatively the geometry and the relative energetics of small neutral and protonated water clusters containing up to five molecules. To correct the total and binding energy for vibrational motion, the zero point energy of the clusters has been calculated by means of the harmonic approximation and by simulating the exact ground state using the diffusion Monte Carlo method. From these $0 \mathrm{~K}$ results, it appears that the anharmonicity accounts for a decrease (increase) of $1.5-5.5 \mathrm{mhartree}(1.0-3.5 \mathrm{kcal} / \mathrm{mol})$ in the total (binding) energy of the protonated clusters. Moreover, we found all the cyclic isomers of $\left(\mathrm{H}_{2} \mathrm{O}\right)_{4} \mathrm{H}^{+}$and $\left(\mathrm{H}_{2} \mathrm{O}\right)_{5} \mathrm{H}^{+}$to be unstable during the diffusion Monte Carlo simulations, and to convert into treelike or linear isomers. Employing the same interaction potential, we also simulated the ground state of $\left(\mathrm{H}_{2} \mathrm{O}\right)_{n}(n=1-5)$ to compute the proton binding energy to a water cluster. This quantity is decreased by roughly $12 \mathrm{mhartree}(7.5 \mathrm{kcal} / \mathrm{mol})$ by including the zero point energy correction to the total energy. The relevance of these findings with respect to the experimental detection and probing of the protonated water clusters is discussed. (C) 2003 American Institute of Physics. [DOI: 10.1063/1.1618222]
\end{abstract}

\section{INTRODUCTION}

During the past few years, attention has been paid to study the proton transfer in both condensed phase and molecular clusters. The motivation for those efforts are many and span various fields of science. Of particular interest are fundamental issues like the unusual large mobility of the proton in water, ${ }^{1}$ the study of the acid dissociation in molecular liquids, ${ }^{2}$ and the acid-base chemistry of protonated water clusters. Unravelling the mechanism of these processes could help in understanding proton conduction through a cell membrane mediated by "water wires," ${ }^{3,4}$ proton mediated electron transfer, ${ }^{5}$ and proton exchange between the environment and the active site of an enzyme. ${ }^{6}$

A common feature shared by all the aforementioned topics is the difficulty of experimentally studying the details of the mechanism of the proton transfer due to the large number of degree of freedom involved. This problem could be circumvented by the use of validated theoretical methods like molecular dynamics or Monte Carlo. Information on the bulk phase can also be inferred by studying the change in the properties of selected systems upon increasing the number of atoms or molecules. Among the mostly intensively studied systems are protonated water clusters, for which infrared spectra and binding enthalpy have been measured. ${ }^{7-12}$ Such experiments highlight the unusual properties of these charged clusters, and among these we cite the large redshift

\footnotetext{
${ }^{a)}$ Electronic mail: Massimo.Mella@chem.ox.ac.uk

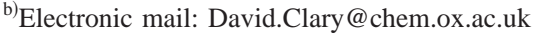

of the $\mathrm{OH}$ stretch due to the presence of the additional $\operatorname{proton}^{7,13,14}$ and the absence of the "cagelike" structures $^{7,9-12}$ characterizing the neutral parents (e.g., see Ref. 15). However, some of these features were inferred by interpreting the experimental results with the help of theoretical modeling ${ }^{7,14,16}$ carried out by means of ab initio calculations or by employing model potentials., ${ }^{3,17,18}$

Notwithstanding the similarity, an important difference exists between the $a b$ initio and model potential approaches, and this is related to the way the nuclear motion is included in the description of a cluster. While ab initio methods customarily assume a well defined structure and introduce nuclear motion as a correction by means of an harmonic expansion of the interaction potential around the minimum, a sensibly chosen model potential may allow description of the overall nuclear motion without introducing such an approximation. This last feature opens the chance to study the possible structural reorganisation that could take place as a function of different thermodynamical variables. ${ }^{13,18}$

It is also true that structural changes could be studied by means of the methodology of "on the fly" molecular dynamics, ${ }^{19}$ where no model analytical form for the interaction is assumed. Here, energy and forces are computed at every step of the simulation using an ab initio or density functional theory (DFT) approach. Although these methods have the advantage of being highly flexible and not requiring the tedious selection of the model potential form and its optimal parameters, they are extremely expensive computationally. ${ }^{4}$ They also do not normally account for 
quantum-dynamical effects on the nuclear motion. The high computational cost of averaging over a large set of system configurations makes it difficult to obtain well converged estimates of the observable. So, accurate model potentials are an appealing alternative to study molecular clusters.

As already pointed out, the most studied protonated species are the protonated water clusters $\left(\mathrm{H}_{2} \mathrm{O}\right)_{n} \mathrm{H}^{+}$. They have been studied theoretically to characterize the equilibrium geometry, ${ }^{16,20-23}$ the proton distribution, ${ }^{3,4}$ and the effect of the temperature. ${ }^{13,18}$ However, few studies have been devoted to quantifying the importance of quantum effects and anharmonicity in the description ${ }^{3}$ of these clusters. Moreover, the main concern of previous studies was to describe the quantum effects in protonated linear chains of water molecules while much less attention has been paid to the cyclic and branched isomers. The branched isomers appear to be the most abundant isomers detected in the infrared spectroscopy experiments. ${ }^{7}$

The interest in studying the quantum effects in these complexes is mainly due to the ground state vibrational energy being larger than the classical thermal energy at $300 \mathrm{~K}$. For instance, a water molecule has a vibrational zero point energy of 0.0225 hartree $(14.1 \mathrm{kcal} / \mathrm{mol})$ (Ref. 23) and an internal thermal energy, as given by the equipartition theorem at $300 \mathrm{~K}$ of $3 k T / 2 \simeq 0.0014$ hartree $(0.87 \mathrm{kcal} / \mathrm{mol})$. This comparison makes apparent that the effect of quantum motion on the total energy is one order of magnitude larger than the thermal one. Since one should expect a similar argument to hold also for larger clusters and maybe for the relative intermolecular motion, this large difference in magnitude could affect significally the behavior of the protonated water clusters. Among the properties that may be affected are the reduction of the "effective" proton transfer barrier height, the classically-forbidden tunnelling between different potential wells, the change in their total binding energy, and in their proton affinity. Moreover, due to the fact that quantum motion should allow the nuclei to visit a wider range of interatomic distances than the thermal one, the anharmonicity of the interaction potential is expected to play a more important role in defining the aforementioned properties when quantum effects are included. Therefore, to model accurately these systems, a more complete understanding of the role played by the quantum effects must be pursued.

With this aim in mind, we decided to study the effects of ground state quantum motion on the energetics and structures of small $\left(\mathrm{H}_{2} \mathrm{O}\right)_{n} \mathrm{H}^{+}$clusters using an all-atom model potential to describe the intra- and intermolecular interactions. The advantage of using a flexible-dissociable-polarizable potential becomes apparent if one recognises that one of the effects of varying the number and location of water molecules in the "proton" solvation shells is changing the chemical nature of the proton bearing entity. More specifically, the first solvation shell decides if the proton is almost equally shared between two molecules (the Zundel $\mathrm{H}_{5} \mathrm{O}_{2}^{+}$cation), or bound to a single molecule. ${ }^{16,21}$ In the last case, the hydronium ion is formed, and it is often solvated by three water molecules accepting hydrogen bonds (the eigen $\mathrm{H}_{9} \mathrm{O}_{4}^{+}$cation). The necessity of describing correctly the relative energy and stability of these two species, rules out the possibility of using rigid-body potentials like the ones presented in Refs. 24-26.

Up to now, many different forms of dissociable potentials have been proposed and implemented to describe the $\left(\mathrm{H}_{2} \mathrm{O}\right)_{n} \mathrm{H}^{+}$family of systems. An early work in this field was made by Stillinger, David, and Weber (SDW), ${ }^{27,28}$ whose potential was built using bare $\mathrm{H}^{+}$and polarizable $\mathrm{O}^{2-}$ ions, and included screening of the Coulomb interaction at short distances. More recently, Ojamae, Singer, and Shavitt ${ }^{21}$ obtained three new model potentials based on an improved SDW form. The most flexible analytical form, dubbed OSS3, contains $\mathrm{O}$ polarizability, dipole-dipole interactions, an effective four-body part, and a better description of the $\mathrm{OH}$ stretch part. The value of the parameters for this potential were obtained by fitting aug-cc-pVTZ MP2 energy results for $\mathrm{H}_{2} \mathrm{O}, \mathrm{H}_{3} \mathrm{O}^{+},\left(\mathrm{H}_{2} \mathrm{O}\right)_{2}$, and $\mathrm{H}_{5} \mathrm{O}_{2}^{+}$. A similar flexible potential was also built by Kozack and Jordan ${ }^{26}$ to study the minima of the protonated water clusters and to account for the possibility of forming a Zundel cation. Here, however, the water molecules are kept rigid at their equilibrium geometry so that the models for both $\mathrm{H}_{3} \mathrm{O}^{+}$and the Zundel cation are not able to reproduce the correct minimum energy structure. In Ref. 21, a detailed summary of the performance of many flexible potentials was also given. There, by extensively comparing many of these model potentials with MP2 and MP4 calculations, the authors reached the conclusions that the OSS series of potentials were the most accurate, among the ones tested, in describing the interaction energy of the small protonated complexes $\mathrm{H}_{3} \mathrm{O}^{+}$and $\mathrm{H}_{5} \mathrm{O}_{2}^{+}$.

As an alternative approach to the modeling of the interaction potential for the protonated water clusters, we mention by the effective valence bond (EVB) method. ${ }^{29}$ This approach, has been recently improved ${ }^{30,31}$ and extended ${ }^{31,32}$ to deal with weak acid-base dissociation, is based on the formal separation of the systems on the basis of valence bond structures coupled together by an effective exchange term. The latter is parameterized to reproduce the energy and geometry of many stationary points on an $a b$ initio (usually MP2) PES. Although easy to parameterize and computationally inexpensive, this model has been shown to suffer from few shortcomings in estimating many-body interactions in the system. This is due to the employed water-water interaction potential. ${ }^{16}$

An important advantage of the OSS3 potential is that it was shown to reproduce with good accuracy both the binding energy and the equilibrium geometry computed at the MP2 level for protonated clusters containing up to four water molecules. Also, although having some difficulty in reproducing the hydrogen bond angle within $4^{\circ}$, OSS 3 was shown to be able to correctly describe the relative energetics and the structure of the $\left(\mathrm{H}_{2} \mathrm{O}\right)_{n}$ clusters $(n=2-5)$. In our view, this is an important element if one wishes to describe larger protonated clusters. Other interesting features of this model potential are its ability to correctly describe the dissociation of a water molecule from the charged cluster, and the dependency on the $\mathrm{O}-\mathrm{O}$ distance of the barrier height for proton exchange in $\mathrm{H}_{5} \mathrm{O}_{2}^{+}$and the linear $\mathrm{H}_{9} \mathrm{O}_{4}^{+}$. For these reasons, we employed the OSS3 model as the interaction potential in all of our calculations.

In this work, we present a study of the structure and 
vibrational motion of the protonated water clusters $\left(\mathrm{H}_{2} \mathrm{O}\right)_{n} \mathrm{H}^{+}(n=1-5)$ using the OSS3 (Ref. 21) interaction potential and the diffusion Monte Carlo (DMC) method to simulate their ground state eigenfunctions. We also computed the ground state energy for neutral water clusters using the same potential to obtain reference values for their proton affinity. In Sec. II we summarize the principal features of the computational method employed in this study. In Sec. III we describe the computational results for the energetics of the clusters, and some of the atom-atom 1D distribution functions sampled by QMC. 2D distribution functions are also presented as a tool to understand the role that quantum effects play in describing the systems. Finally, Sec. IV contains our conclusions.

\section{QUANTUM MONTE CARLO METHODS}

Since quantum Monte Carlo methods are already well described in the literature, ${ }^{33}$ it is not necessary to present the diffusion Monte Carlo technique in detail. Here, we simply introduce its relevant features, and present only the technical details needed for a complete understanding of our computational results.

An imaginary-time version of the Schrödinger equation serves as a starting point to develop the theory of the DMC methods. ${ }^{34}$ Specifically, this reads

$$
\frac{\partial \Psi(\mathbf{R}, t)}{\partial t}=\sum_{i}^{N} \frac{\nabla_{i}^{2}}{2 m_{i}} \Psi(\mathbf{R}, t)-\left[V(\mathbf{R})-E_{\mathrm{ref}}\right] \Psi(\mathbf{R}, t),
$$

where $V(\mathbf{R})$ is the interaction potential, and $\mathbf{R}$ is a point in $3 N$ space.

Although we leave the analytical form of the interaction potential undefined as a way to stress the ability of Monte Carlo to cope with any local potential, we point out that the quality, accuracy and stability of the method strongly depend on the specific potential.

For the nodeless function case we consider in this work (i.e., notwithstanding the fermionic character of the $\mathrm{H}$ atoms, we assume that all the particles in our systems obey Boltzmann's statistics), the previous equation is formally equivalent to a classic diffusion process with sink and source terms dependent on the position in space. Here, the assumption of Boltzmann's statistics is justified by the fact that the $\mathrm{H}$ atoms do not exchange during the simulations, so that the effect of the spin statistics is expected to be negligible.

Equation (1) can be recast in the integral form,

$$
\begin{aligned}
\Psi\left(\mathbf{R}^{\prime}, t+d t\right) & =\int d \mathbf{R} G\left(\mathbf{R} \rightarrow \mathbf{R}^{\prime}, d t\right) \Psi(\mathbf{R}, t) \\
& =\sum_{j} c_{j} e^{-(t+d t)\left(E_{j}-E_{\mathrm{ref}}\right)} \psi_{j}(\mathbf{R}),
\end{aligned}
$$

where $G\left(\mathbf{R} \rightarrow \mathbf{R}^{\prime}, d t\right)=\left\langle\mathbf{R}^{\prime}\left|e^{-d t H}\right| \mathbf{R}\right\rangle$ is the imaginary time Green's function, and $c_{j}=\int \Psi(\mathbf{R}, 0) \psi_{j}(\mathbf{R}) d \mathbf{R}$. If this function were known, Monte Carlo methods could be used to sample the above integral projecting out all the excited state components from the starting wave function $\Psi(\mathbf{R}, 0) .{ }^{33}$ In this case the technique would sample a distribution proportional to the exact ground state eigenfunction $\Psi_{0}(\mathbf{R})$.
Unfortunately, the exact Green's function is known only for very simple model Hamiltonians. For systems whose ground state wave function has no nodes, the theory of DMC relies completely on the ability to find an accurate approximation to the imaginary time Green's function of the Schrödinger equation. The usual short time approximation (STA) to this function is given by the Trotter formula $(T),{ }^{35}$

$$
\begin{aligned}
G_{\mathrm{STA}}^{T}\left(\mathrm{R} \rightarrow \mathrm{R}^{\prime}, d t\right) \\
=\prod_{i}\left(\frac{m_{i}}{2 \pi d t}\right)^{3 / 2} \exp \left[-\frac{m_{i}\left(\mathbf{r}_{i}^{\prime}-\mathbf{r}_{i}\right)^{2}}{2 d t}\right] \\
\\
\times \exp \left[-d t \frac{V(\mathbf{R})+V\left(\mathbf{R}^{\prime}\right)-2 E_{\mathrm{ref}}}{2}\right]
\end{aligned}
$$

and is accurate to a second order in $d t$. That is, for short time steps, the error is proportional to $d t^{3}$. This equation is commonly simulated by interpreting the normalized Gaussian kernel as a transition matrix, while the $\exp \left\{-d t\left[V\left(\mathbf{R}^{\prime}\right)\right.\right.$ $+V(\mathbf{R})] / 2$ \} part is simulated by means of a discrete birth/ death branching process of the walker population.

To improve the efficiency of the DMC method, importance sampling (IS) is usually introduced in the simulation by using a trial wave function $\Psi_{T}$. So, DMC samples $f(\mathbf{R}, t)=\Psi_{T}(\mathbf{R}) \psi_{0}(\mathbf{R})$ instead of $\psi_{0}(\mathbf{R})$. This is done to reduce the statistical error of the energy results, to speed up the convergence of the simulation by avoiding uninteresting regions of the configurational space, and to restrain a weakly bound system from dissociating during the projecting evolution. However, biasing a priori the sampling by a $\Psi_{T}$ may increase the chance of missing regions of the configurational space that are important for the accurate description of structural properties of the system. So, in this work, and for computational simplicity, we restricted ourselves to use a DMC version without the IS procedure.

Because of our choice to use Eq. (3) without IS, the DMC method samples the ground state $\Psi_{0}$ of the system. Hence, in order to compute the total energy of the cluster, we use the potential energy estimator introduced by Anderson ${ }^{34}$

$$
\left\langle E_{V}(t)\right\rangle=\frac{\int \Psi_{0}(\mathbf{R}) V(\mathbf{R}) d \mathbf{R}}{\int \Psi_{0}(\mathbf{R}) d \mathbf{R}}
$$

that converges to the ground state energy for $d t \rightarrow 0$. This estimator has been shown to behave smoothly for many different model and realistic systems, ${ }^{36,37}$ and to have a reasonably large time step range in which it follows a quadratic dependency on the time step. This allows either extrapolation to $d t=0$, or the selection of a time step $d t$ short enough to ensure that the time step bias is smaller than the statistical error of the energy mean values.

More accurate STA than Eq. (3) have been proposed ${ }^{36-38}$ and tested in DMC calculations. However, our test runs on the smaller protonated water clusters indicate that it is possible to obtain easily the needed accuracy and statistical precision with the simple Trotter factorization, so we restrict ourself to use Eqs. (3) and (4) as our working equations.

As a final point about the simulation algorithm, it is important to stress that any version of the DMC method employing the branching algorithm suffers from a population 


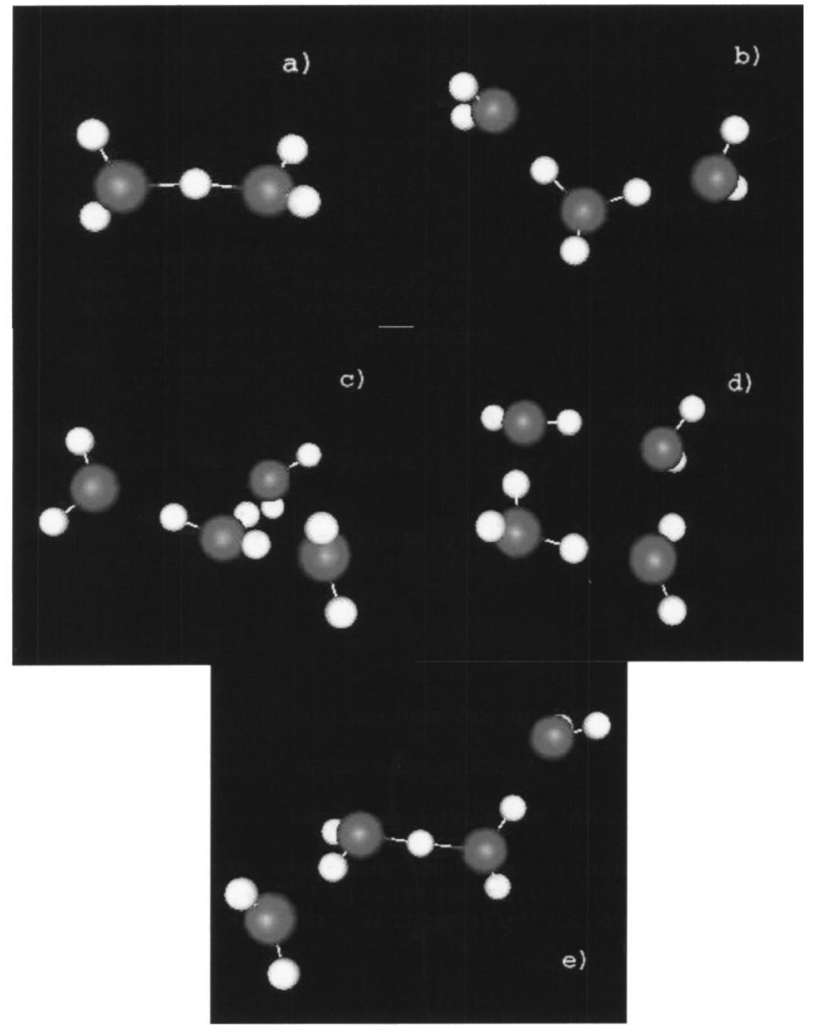

FIG. 1. Optimized OSS3 structures for (a) $\mathrm{H}_{5} \mathrm{O}_{2}^{+}$, (b) $\mathrm{H}_{7} \mathrm{O}_{3}^{+}$, (c) branched $\mathrm{H}_{9} \mathrm{O}_{4}^{+} 4^{(1)}$, (d) cyclic $\mathrm{H}_{9} \mathrm{O}_{4}^{+} 4^{(2)}$, and (e) linear $\mathrm{H}_{9} \mathrm{O}_{4}^{+} 4^{(3)}$.

control bias due the fluctuation of $E_{\text {ref }} \cdot{ }^{39}$ This quantity is constantly updated to keep the configuration ensemble around a chosen target population $M,{ }^{34}$ and this procedure has been shown to introduce a bias in the energy proportional to $1 / M$ if $M$ is large enough. Although many useful procedures have been devised to overcome this difficulty, in this work we employed the simple approach of running two simulations with different numbers of walkers in the range of $M$ where the dependency of $E_{V}$ can be considered linear. As we will show, the two energy values allow us to safely extrapolate to infinite population and to obtain unbiased results.

\section{SIMULATIONS AND RESULTS}

As a starting point for all our DMC simulations of the $\left(\mathrm{H}_{2} \mathrm{O}\right)_{n} \mathrm{H}^{+}$clusters, we used the minima obtained by Hodges and Wales ${ }^{40}$ employing the Hodges and Stone ASP potential energy surface. ${ }^{24}$ As pointed out previously, this interaction potential is built considering the cluster partitioned in $\mathrm{H}_{2} \mathrm{O}$ and $\mathrm{H}_{3} \mathrm{O}^{+}$fragments that are kept frozen in their own minimum geometry. So, in order to have a meaningful comparison between total energy of different isomers, and to make sure that a specific isomer exists also on the OSS3 surface, we reoptimized every cluster using Powell's method with a tight convergency criterion. The resulting geometries are shown in Figs. 1 and 2 while the OSS3 energy of the optimized clusters are reported in Table I. There, we use the notation $k^{(l)}$ to indicate the $l$ th isomer of the cluster containing $k$ water molecules. The isomers are ordered following the increase of total energy. We found that all the reoptimized

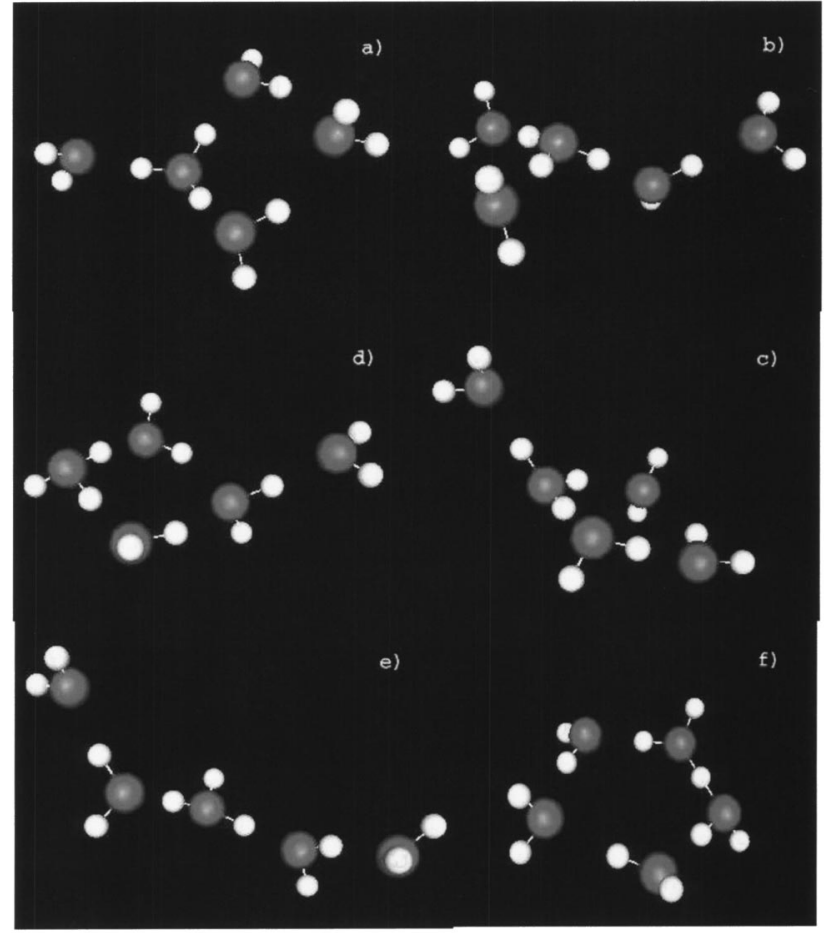

FIG. 2. Optimized OSS3 structures for the $\mathrm{H}_{11} \mathrm{O}_{5}^{+}$isomers. (a) Kitelike $5^{(1)}$, (b) branched $5^{(2)}$, (c) and (d) cyclic $5^{(3)}$ and $5^{(4)}$, (e) linear $5^{(6)}$, (f) penthagonal $5^{(5)}$

isomers remained stable, though they undergo minor changes in the atomic distances and angles. The largest changes were seen optimizing the $\left(\mathrm{H}_{2} \mathrm{O}\right)_{2} \mathrm{H}^{+}$cluster and the parent of the linear isomer $4^{(3)}$. These changes were due to the migration of one of the $\mathrm{H}_{3} \mathrm{O}^{+}$protons towards the center of the water chain. This proton displacement gave rise to a more symmetric system where it is equally shared between the two central water molecules. All of the optimized cyclic complexes have at least one double acceptor water molecule in the ring. This is probably due to the need of the hydronium moiety to donate two $\mathrm{H}$ bonds to stabilize itself. This is different from what happens in the pure water clusters with $n \leqslant 5$, where the most stable ring isomers always have water molecules acting as donor-acceptor. ${ }^{41}$

Interestingly, no chainlike isomer was found among the ASP-optimized pentamers. ${ }^{40}$ Since this is expected to exist on the basis of the MP2 and B3LYP calculation carried out by Christie and Jordan, ${ }^{16}$ we examined some possible global minimum candidates by simply adding another water molecule to the optimized linear tetramer. At the end of the optimization, we found in all our candidates that the proton moved closer to the central water molecule reforming the $\mathrm{H}_{3} \mathrm{O}^{+}$entity as predicted by the $a b$ initio calculations. ${ }^{16}$ From this set of optimized geometries, we extracted the lowest energy one as our global minimum conformer for this isomer.

Since the minima of Ref. 40 were obtained by using the ASP potential, ${ }^{24}$ it could be possible to have additional local minima on the OSS3 surface used in this work not contained in the set presented in Ref. 40. To test this possibility, we used the approach proposed by Finnila et $a l^{42}$ and run long 
TABLE I. OSS3 energy minimum $\left(V_{\min }\right)$, DMC ground state energy $\left(E_{0}(n)\right)$, zero point energy (ZPE), and grow energy $\left(E_{\text {grow }}\right)$ for the $\left(\mathrm{H}_{2} \mathrm{O}\right)_{n} \mathrm{H}^{+}$systems as a function of $n$. The ZPE is estimated using both DMC simulations and the harmonic oscillator (HA) approximation. The DMC results are extrapolated to infinite walker population. The double entry for $E_{\text {grow }}$ of $5^{(2)}$ is due to its possible formation from $4^{(1)}$ or $4^{(3)}$. Quantities in a.u. apart from the $E_{\text {grow }}$ values between square brackets that are expressed in $\mathrm{kcal} / \mathrm{mol}$.

\begin{tabular}{lccccc}
\hline \hline$n$ & $V_{\min }$ & $E(n)_{0}$ & ZPE & $E_{\text {grow }}$ & ZPE(HA) \\
\hline 1 & -1.515342 & $-1.482253(7)$ & $0.033089(7)$ & $0.15595(2)[97.79(1)]$ & 0.034517 \\
2 & -2.917622 & $-2.85862(3)$ & $0.05900(3)$ & $0.05008(3)[31.41(2)]$ & 0.061197 \\
3 & -4.303191 & $-4.21879(4)$ & $0.08440(4)$ & $0.03388(4)[21.25(2)]$ & 0.088060 \\
$4^{(1)}$ & -5.681306 & $-5.57188(9)$ & $0.10942(9)$ & $0.02680(10)[16.81(6)]$ & 0.112665 \\
$4^{(2)}$ & -5.677980 & R. O. & & & 0.114540 \\
$4^{(3)}$ & -5.677913 & $-5.56962(9)$ & $0.10829(9)$ & $0.02454(10)[15.39(6)]$ & 0.112868 \\
$5^{(1)}$ & -7.052958 & R. O. & & & 0.138955 \\
$5^{(2)}$ & -7.051326 & $-6.9165(1)$ & $0.1348(1)$ & $0.01833(16)[11.5(1)]$ & 0.138120 \\
& & & & $0.02059(16)[12.9(1)]$ & \\
$5^{(3)}$ & -7.049961 & R. O. & & & 0.139144 \\
$5^{(4)}$ & -7.049823 & R. O. & & & 0.139708 \\
$5^{(5)}$ & -7.049226 & R. O. & & & 0.139217 \\
$5^{(6)}$ & -7.048409 & $-6.9156(2)$ & $0.1328(2)$ & $0.01954(20)[12.3(1)]$ & 0.138354 \\
\hline
\end{tabular}

DMC simulations employing atomic masses 10-20 times smaller than the correct ones. This has been shown to enhance the quantum effects of the system, and therefore to enhance the sampling of the configurational space. During each DMC simulation, a subset of walkers was randomly extracted from the total population and saved on a file. The ensamble of collected walkers was successively optimized by minimizing their potential energy, and the optimized structures were compared with the set of geometries obtained in Ref. 40. No additional low energy isomers were found as result of this procedure.

Before discussing our results in detail, it is worth pointing out that some of the isomers obtained by basin-hopping optimization, ${ }^{40}$ and hence also our optimized ones, differ simply by the relative torsional orientation of the hydrogen atoms not involved in forming hydrogen bonds. We dub these sets of similar isomers "free-H conformers." The different relative orientation for the $\mathrm{H}$ atoms accounts for a difference in total energy of roughly $1.5-3$ mhartree $(0.94-$ $1.88 \mathrm{kcal} / \mathrm{mol}$ ), in both the ASP and our OSS3 results. This energy difference indicates the probability that the free- $\mathrm{H}$ conformers could convert, overcoming the barriers separating them as an effect of the quantum zero point motion during the DMC simulation. This was checked by visualizing the structural changes in walker ensemble, and we found that all the possible free- $\mathrm{H}$ conformers were represented in it. As a consequence, we simply report the total energy and simulation results for the lowest energy free-H conformer of any given isomer.

Beginning with optimized geometries, all the simulations were carried out in two stages. In the first equilibration stage, two to five hundred identical walkers were created and successively displaced and branched following the rules of the DMC algorithm. ${ }^{34}$ A short time step was employed to avoid the possibility of having a walker ensemble collapsing into a single representative configuration due to the initially large fluctuations of their weights. Once the configuration set has reached the equilibrium, we gradually incremented the total population using a time step suitable for a production run. The target population was usually composed of 2000 or 4000 walkers. As a second stage, we carried out long production runs, each one composed of, at least, 200 blocks of 500 DMC steps each. So, while our shortest simulations sampled a total of at least $2 \times 10^{8}$ configurations, the average one was composed by roughly $1 \times 10^{9}$ samples. At the end of each simulation, the blocking procedure ${ }^{43}$ was used to test for the presence of residual serial correlation and to obtain a more accurate estimate of the standard error of the mean values. This procedure amounts to recursively "block" together two by two the simulation data and to compute the standard error of the average using the new set of "blocked" data. It can be proved that the estimated standard error converges to its unbiased value when the serial correlation between successive "blocked" data reduces to zero.

In order to obtain some insight on the time step and population bias for these systems, we run simulations using various numbers of walkers and time steps on the $\mathrm{H}_{5} \mathrm{O}_{2}^{+}$ system. For these test runs, the less accurate, but computationally less expensive, OSS2 potential was used instead of the OSS3 one. The total energy results obtained by simulating $\mathrm{H}_{5} \mathrm{O}_{2}^{+}$with the OSS2 model, with 2000 walkers, and time steps ranging from 2 to 40 a.u. are shown in Fig. 3. This picture clearly shows that in the range $2-20$ a.u., the average DMC energy obtained by means of the Trotter approximation Eq. (3) has a neat quadratic behavior. This fact is indicated by the excellent agreement between the computed values and the fitted $a+b * d t^{2}$ form (dashed line) in the same time step range. Moreover, comparing the extrapolated energy value at $d t=0$ with the one computed at $d t=2$, respectively, $-2.90192(4)$ and $-2.90191(3)$ hartree, it emerges that they are statistically indistinguishable. In our view, this indicates that $d t=2$ could be safely used to run all the simulations without the necessity of extrapolating to infinitesimally short $d t$. As for the population bias, Fig. 4 shows the OSS2 total energy of $\mathrm{H}_{5} \mathrm{O}_{2}^{+}$computed using $d t=2$ versus the reciprocal of the number of walkers, $1 / M$. Here, the theoretically predicted linear behavior ${ }^{39}$ is clearly seen for all the $M$ values, 


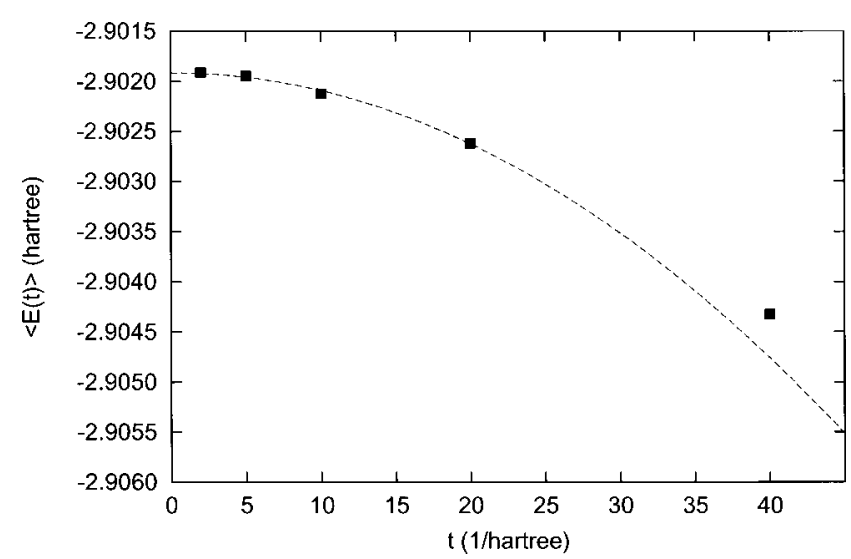

FIG. 3. DMC energy for $\mathrm{H}_{5} \mathrm{O}_{2}^{+}$as a function of the time step $t$. The OSS2 model is used in the simulations. Quantities in a.u.

namely 500, 1000, 2000, and 4000 walkers. This plot also indicates that the idea of linearly extrapolating the energy to an infinite number of walkers using only two different target populations may be posed on solid grounds. To test this hypothesis, we built all the possible subsets composed only by two $\left[\left(d t_{1}, E_{1}\right)\right.$ and $\left.\left(d t_{2}, E_{2}\right)\right]$ of the data shown in Fig. 4. Using each of these subsets, we obtained the parameters of the stright line which interpolates them, and successively estimated the statistical error of the intersection of this straight line with the $y$ axis. This was done by generating thousands of couples of $\left(d t_{1}, E_{1}\right)$ and $\left(d t_{2}, E_{2}\right)$ points by means of a short Monte Carlo simulation. Here, two Gaussian distributions of energy values having the same mean value and a standard deviation equal to the standard error of the two fitted data were sampled. For each couple of sampled points, we reestimated the intersection and collected it to produce a standard error. All the two-point estimates so obtained were found to differ by no more than twice the standard error of the estimate obtained using the complete data set. So, the different estimates are statistically indistinguishable, and therefore supporting our idea of extrapolating to a infinite target population of walkers.

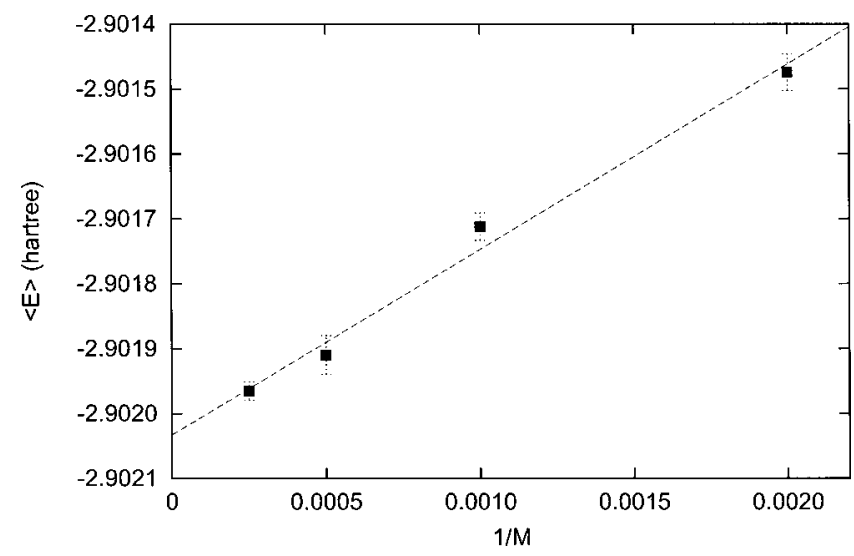

FIG. 4. DMC energy for $\mathrm{H}_{5} \mathrm{O}_{2}^{+}$as a function of the inverse of number $M$ of walkers in the population. The OSS2 model is used in the simulations. Quantities in a.u.

\section{A. Energetics}

The values of the total energy $E_{0}$ for the various protonated and neutral clusters obtained by means of the DMC simulations are shown in Tables I and II, respectively. In the same tables, we also present the zero point energy (ZPE) contribution to this value as computed by means of the formula $\mathrm{ZPE}=V_{\min }-E_{0}$. For $\left(\mathrm{H}_{2} \mathrm{O}\right)_{n} \mathrm{H}^{+}$, we also report the differential quantity,

$$
E_{\text {grow }}^{a b}(n)=E_{0}^{a}(n-1)-E_{0}^{b}(n),
$$

where $E_{0}^{a}(n)$ is the total energy of the $a$ isomer of the protonated cluster containing $n$ water molecules. $E_{\text {grow }}(1)$ is the proton affinity of the water molecule. $E_{\text {grow }}^{a b}(n)$ can be interpreted as the vaporization energy of a water molecule from the cluster which depends on the structure of both the $a$ and $b$ isomers as well as on the total number $n$ of water molecules. An analogous quantity has been computed also for the neutral water clusters and is shown in Table II.

Before we begin discussing the behavior of the energy quantities, it is worth pointing out that, in Table I, the entry "R.O." indicates that the particular cyclic isomer studied was found to convert by ring opening (R.O.) during the DMC simulations. Conversely, all the linear and treelike isomers for the different clusters never showed any propensity to change their structure [apart from the changes in torsional angles in $4^{(2)}, 5^{(2)}$, and $5^{(6)}$ ] or the relative connectivity of the oxygen atoms. This last fact allows us to interprete many of the results on the basis of the geometry of minimum potential energy structure of the $\mathrm{O}$ atoms.

The ZPE and $E_{\text {grow }}^{a b}(n)$ for $\left(\mathrm{H}_{2} \mathrm{O}\right)_{n} \mathrm{H}^{+}$are also shown in Fig. 5. As to $E_{\text {grow }}^{a b}(n)$, the trend clearly indicates a decrease of this quantity upon increasing the number of water molecules in the cluster. Although too small to be distinguished in the scale of Fig. 5, both the tetramer and the pentamer are represented by two and three data points, respectively. As indicated previously, this is due to the different isomers of $\left(\mathrm{H}_{2} \mathrm{O}\right)_{4} \mathrm{H}^{+}$and $\left(\mathrm{H}_{2} \mathrm{O}\right)_{5} \mathrm{H}^{+}$, and by the fact that isomer $5^{(2)}$ can be built starting either from $4^{(1)}$ or $4^{(3)}$ by adding a water molecule. The decreasing trend of $E_{\text {grow }}^{a b}(n)$ can be rationalized in terms of the many-body effects due to the electrostatic interaction. Specifically, besides polarizing the surrounding molecules, the net excess charge due to the proton tends to align the water molecules with an unfavorable relative orientation of their dipole moments (for instance, see the trimer clusters in Fig. 1). This reduces the incremental interaction energy of a single water molecule going from the dimer to the trimer and finally to the tetramer. For the pentamer, the fifth water molecule is at longer distance from the charge bearing entity (i.e., it is in the "second" solvation shell) so it feels a more distant and shielded positive charge. Notwithstanding this, the binding energy for the fifth water molecule still has a value spanning the range 0.01693(16) $0.02047(16)$ hartree $[10.6(1)-12.8(1) \mathrm{kcal} / \mathrm{mol}]$ which is larger than the corresponding quantity for the neutral water pentamer [0.0118(2) hartree or 7.4(1) kcal/mol, see Table II].

Figure 5 also shows the values for the ZPE for all the protonated clusters. This quantity follows a linear behavior with respect to the number of water molecules present in the 
TABLE II. OSS 3 energetics of the $\left(\mathrm{H}_{2} \mathrm{O}\right)_{n}$ systems as a function of $n$. The meaning of the symbols is identical to the caption of Table I. The DMC results are extrapolated to infinite walker population. Quantities in a.u. apart from the $E_{\text {grow }}$ values between square brackets that are expressed in $\mathrm{kcal} / \mathrm{mol}$.

\begin{tabular}{cccccc}
\hline \hline$n$ & $V_{\min }$ & $E(n)_{0}$ & ZPE & $E_{\text {grow }}$ & ZPE(HA) \\
\hline 1 & -1.348285 & $-1.326290(5)$ & $0.021995(5)$ & & 0.022536 \\
2 & -2.705378 & $-2.658849(8)$ & $0.046529(8)$ & $0.006269(7)[3.931(4)]$ & 0.047845 \\
3 & -4.072900 & $-4.00043(2)$ & $0.07247(2)$ & $0.015093(11)[9.465(6)]$ & 0.074403 \\
4 & -5.444560 & $-5.34680(6)$ & $0.0977(10)$ & $0.02008(6)[12.59(3)]$ & 0.100030 \\
5 & -6.808804 & $-6.6866(2)$ & $0.1222(2)$ & $0.0135(2)[8.47(1)]$ & 0.124944 \\
\hline \hline
\end{tabular}

cluster. The deviation from the linear approximation has been found to be always smaller than 0.001 hartree (roughly $220 \mathrm{~cm}^{-1}$ ), and we suspect the linear behavior to be mostly due to the linear increase of $\mathrm{OH}$ bonds and to the fact that the coupling between different $\mathrm{OH}$ stretches is usually small. ${ }^{3}$

Comparing the ZPE computed by means of DMC for the stable isomers with the same quantity obtained using the simpler harmonic oscillator approximation [ZPE(HA), Table I], it appears that the anharmonicity accounts for a decrease in total energy of $1.5-5.5 \mathrm{mhartree}(0.9-3.4 \mathrm{kcal} / \mathrm{mol})$. The largest variations are obtained for the trimer (3.6 mhartree or $2.3 \mathrm{kcal} / \mathrm{mol})$, the linear tetramer $4^{(3)}(4.6$ mhartree or 2.9 $\mathrm{kcal} / \mathrm{mol})$, and the linear pentamer $(5.5$ mhartree, $3.4 \mathrm{kcal} /$ mol). This finding could be explained by the same features of these three systems, namely a strong anharmonicity in the potential energy surface for the proton exchange in the central $\mathrm{H}_{5} \mathrm{O}_{2}^{+}$part of the linear tetramer, and the large amplitude motion of the $\mathrm{H}$ bonded excess proton connected to the central water molecule in the trimer and linear pentamer.

In order to assess the accuracy of the results obtained by means of the model OSS3 potential, it is interesting to compare different methods. For this reason, Table III shows the adiabatic formation energy developed during the process,

$$
(n-1) \mathrm{H}_{2} \mathrm{O}+\mathrm{H}_{3} \mathrm{O}^{+} \rightarrow\left(\mathrm{H}_{2} \mathrm{O}\right)_{n} \mathrm{H}^{+}
$$

for the isomers that were found to be stable during the DMC simulations. This quantity is computed using $\operatorname{OSS} 3\left(\Delta V_{\min }\right)$, MP2, DFT-B3LYP, and the MSEVB model potentials. ${ }^{16,44}$ We also report in Table III the difference between the ground state DMC $E_{0}$ results $\left(\Delta E_{0}\right)$ for the same process, and the

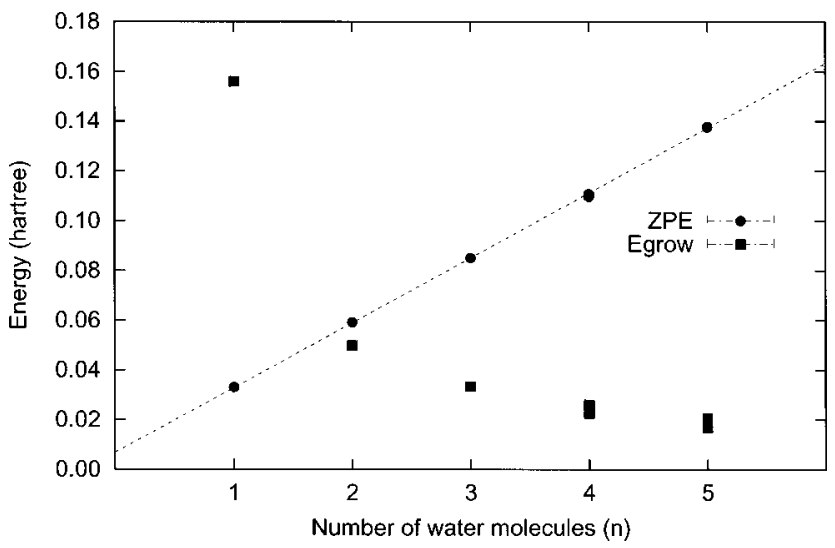

FIG. 5. DMC zero point energy (ZPE) and grow energy ( $\left.E_{\text {grow }}\right)$ of $\left(\mathrm{H}_{2} \mathrm{O}\right)_{n} \mathrm{H}^{+}$as a function of $n$. The dashed line is a linear fit of the ZPE values. Quantities in a.u. difference $\Delta \mathrm{ZPE}=\Delta E_{0}-\Delta V_{\min } \Delta \mathrm{ZPE}$ represents the correction to the adiabatic formation energy due to the vibrational motion.

For the sake of comparison, we use the MP2 results as reference for all the other methods. On the basis of the known performance of MP2 with a large basis set, ${ }^{16,21}$ these are expected to be close to the exact values. However, MP4 calculations on the same systems show the tendency to slightly reduce both total and formation energy. ${ }^{21}$

Comparing the OSS3 and the MP2 results, it appears that the model potential is fairly accurate in reproducing the formation energy for all the stable clusters. The most notable discrepancies are found for the isomer $5^{(2)}$, whose formation energy is underestimated by $3.5 \mathrm{mhartree}(2.2 \mathrm{kcal} / \mathrm{mol})$. OSS3 works rather well for the trimer and the linear isomers $4^{(3)}$ and $5^{(6)}$.

Since a more thorough comparison of the relative performance of MP2 and B3LYP has already been given in Ref. 16 , we restrict our comment only to the stable isomers. From Table III one can notice that B3LYP overestimates by roughly 2 mhartree $(1.2 \mathrm{kcal} / \mathrm{mol})$ the MP2 values for the smallest clusters but performs accurately for the two pentamers. The MSEVB potential performs rather poorly in terms of the formation energy for $4^{(1)}$ and $5^{(6)}$, and as is pointed out in Ref. 16, this outcome seems to be related to the use of the TIP3P potential for describing the intermolecular water interactions. Considering the results for OSS3 and MSEVB (Table III) together with the extensive comparison carried out in Ref. 21, we conclude that the OSS3 potential could be considered the best flexible polarizable potential available for simulating small protonated water clusters.

Table III shows that the ZPE correction has a profound influence on the absolute value of the formation energy, reducing it by an amount that increases with the size of the cluster. The decrease is found to vary in the range between 4.0 mhartree $(2.5 \mathrm{kcal} / \mathrm{mol})$ for the dimer, and 13.4 mhartree $(8.4 \mathrm{kcal} / \mathrm{mol})$ for the $5^{(2)}$ pentamer. Interestingly, both the linear isomer of the tetramer and the pentamer show a smaller ZPE correction than the corresponding branched isomers. Since the latter display values of the $\mathrm{ZPE}(\mathrm{HA})$ very similar to the linear counterparts, as shown in Table I, this effect should be entirely due to the anharmonicity of the vibrational motion. If we assume that a similar correction is also present for larger clusters and at higher temperatures (e.g., $170 \mathrm{~K}$, the estimated cluster temperature in the experiments described in Ref. 7), this should change the relative stabilities between the branched $\left(\mathrm{H}_{2} \mathrm{O}\right)_{n} \mathrm{H}^{+}$and the linear 
TABLE III. Formation energy of the $\left(\mathrm{H}_{2} \mathrm{O}\right)_{n} \mathrm{H}^{+}$systems as a function of $n$. $V_{\text {min }}$ refers to OSS3 results, $\Delta E_{0}$ is given by DMC energy differences, and $\Delta \mathrm{ZPE}=\Delta E_{0}-V_{\min }$. MP2, B3LYP, and MSEVB pentamer results are taken from Refs. 16, and 44. The MSEVB results for the dimer, trimer, and branched tetramer $4^{(1)}$ are from Ref. 31. The DMC results are extrapolated to infinite walker population. Quantities in a.u.

\begin{tabular}{lcccccc}
\hline \hline$n$ & $V_{\min }$ & $\Delta E_{0}$ & $\Delta$ ZPE & MP2 & MSEVB & \multirow{2}{*}{ B3LYP } \\
\hline 2 & 0.053995 & $0.05008(3)$ & $0.00392(2)$ & 0.05477 & 0.05396 & \\
3 & 0.091279 & $0.08396(3)$ & $0.00731(3)$ & 0.09175 & 0.09110 & \\
$4^{(1)}$ & 0.121109 & $0.11076(9)$ & $0.01035(9)$ & 0.1233 & 0.12690 & 0.1249 \\
$4^{(3)}$ & 0.117716 & $0.10850(9)$ & $0.00922(9)$ & 0.1178 & & 0.1197 \\
$5^{(2)}$ & 0.142844 & $0.1291(1)$ & $0.0137(1)$ & 0.1463 & 0.14682 & 0.146183 \\
$5^{(6)}$ & 0.139927 & $0.1282(2)$ & $0.0117(2)$ & 0.1411 & 0.13390 & 0.141543 \\
\hline \hline
\end{tabular}

$\left(\mathrm{H}_{2} \mathrm{O}\right)_{(n-1)} \mathrm{H}^{+}$by roughly $3-4$ mhartree (i.e., roughly 2.5 $\mathrm{kcal} / \mathrm{mol})$. The same quantity computed employing the harmonic approximation ZPE(HA) values from Table I and Table II is in quite good agreement with $\triangle \mathrm{ZPE}$ for the dimer, the trimer, and the branched tetramer and pentamer. However, it is larger by more than 1.5 mhartree $(0.9 \mathrm{kcal} / \mathrm{mol})$ for the linear tetramer and pentamer. This last outcome is mostly a by-product of the anharmonicity of the linear isomer vibrational motion with respect to the branched one.

Another interesting energetic quantity is the proton affinity of a water cluster. This is the energy released during the process

$$
\left(\mathrm{H}_{2} \mathrm{O}\right)_{n}+\mathrm{H}^{+} \rightarrow\left(\mathrm{H}_{2} \mathrm{O}\right)_{n} \mathrm{H}^{+},
$$

where the neutral water cluster is assumed to be described by the isomer with the lowest vibrational ground state energy $E_{0}$. These are the cyclic isomers where all the water molecules act as donor-acceptors. The proton affinity is expected to depend on the number of water molecules present in the parent neutral clusters due to the reorientation and solvation processes that take place after adding the proton. Our results for this quantity, computed using the minimum potential energy and shown in Table IV, indicate that this is exactly the case, going from 0.167 hartree $(104 \mathrm{kcal} / \mathrm{mol})$ for the formation of $\mathrm{H}_{3} \mathrm{O}^{+}$to roughly 0.240 hartree $(151 \mathrm{kcal} /$ mol) for the proton addition to the two pentamers. A similar behavior is found also for the proton affinity computed using the $E_{0}$ results. Moreover, using the values of the proton affinity (PA) and of the binding energy (BE) in Table III, one can estimate the energy released during the process,

$$
\left(\mathrm{H}_{2} \mathrm{O}\right)_{n}+\mathrm{H}^{+} \rightarrow\left[\left(\mathrm{H}_{2} \mathrm{O}\right)_{n} \mathrm{H}^{+}\right]^{*} \rightarrow(n-1) \mathrm{H}_{2} \mathrm{O}+\mathrm{H}_{3} \mathrm{O}^{+}
$$

TABLE IV. OSS3 proton affinity of the $\left(\mathrm{H}_{2} \mathrm{O}\right)_{n}$ clusters as a function of $n$. The results are extrapolated to an infinite walker population. Quantities in a.u. apart from the $\Delta E(n)_{0}$ values between square brackets that are expressed in $\mathrm{kcal} / \mathrm{mol}$.

\begin{tabular}{lccc}
\hline \hline$n$ & $\Delta V_{\min }$ & $\Delta E(n)_{0}$ & $\Delta \mathrm{ZPE}$ \\
\hline 1 & 0.167057 & $0.1555963(9)[97.803(6)]$ & $0.011138(9)$ \\
2 & 0.212244 & $0.19982(2)[125.31(1)]$ & $0.01261(2)$ \\
3 & 0.230291 & $0.21860(3)[137.08(2)]$ & $0.01211(3)$ \\
$4^{(1)}$ & 0.236746 & $0.2251(1)[141.16(6)]$ & $0.0111(1)$ \\
$4^{(3)}$ & 0.233353 & $0.2228(1)[139.72(6)]$ & $0.0112(1)$ \\
$5^{(2)}$ & 0.242522 & $0.2299(2)[144.2(1)]$ & $0.0116(2)$ \\
$5^{(6)}$ & 0.239605 & $0.2290(2)[143.6(1)]$ & $0.0114(2)$ \\
\hline \hline
\end{tabular}

which is given by PA-BE. In Eq. (8) $\left[\left(\mathrm{H}_{2} \mathrm{O}\right)_{n} \mathrm{H}^{+}\right]^{*}$ indicates a vibrationally excited protonated cluster. At this point it is worth remembering that the ground state of $\left(\mathrm{H}_{2} \mathrm{O}\right)_{n} \mathrm{H}^{+}$ is stable with respect to the dissociation into $(n-1) \mathrm{H}_{2} \mathrm{O}$ and $\mathrm{H}_{3} \mathrm{O}^{+}$, i.e., this process has a positive energy change. Instead, $\left[\left(\mathrm{H}_{2} \mathrm{O}\right)_{n} \mathrm{H}^{+}\right]^{*}$ is generated if the energy released during the proton addition is stored in the system and not dissipated by means of a collision with another molecule. Since PA-BE is positive for the studied systems, it is clear that all of the clusters may fragment after the addition of the proton.

Interestingly, the ZPE correction to the proton affinity appears to level off around 0.011 hartree $(6.9 \mathrm{kcal} / \mathrm{mol})$ after $n=4$. At the present, we do not have a clear explanation for this observation, but it seems to indicate that the excess proton introduces roughly the same ZPE change disregarding the chemical environment.

Finally, it is noted that computing the difference between the DMC results and the one obtained by using the harmonic approximation is just one way to explore the effects of the anharmonicity in the ground state of a system. As was recently highlighted, ${ }^{45}$ a small difference between these two quantities could mask a much larger anharmonic behavior due to two opposite effects. Those effects are the steep increase of the energy in the repulsive region of the potential for $r_{i j} \rightarrow 0$ and the shallow wall for $r_{i j}>r_{\text {eq }}$. Whereas the first effect would more strongly localise the wave function of the system, and increase both the average kinetic and potential energies, the second would allow the ground state wave function to extend to larger distance than in the harmonic case. This can produce a partial cancellation of the effect of the anharmonicity in the computed total energy.

\section{B. Structure}

Being the lighter element, the hydrogen atom is well known to show interesting quantum effects on many properties. Its large amplitude motion allows it to sample wide regions of the PES and enables quantum mechanical tunnelling between two separated wells if the potential energy barrier is not too high or wide. This can be seen as a manifestation of the breakdown of the second order approximation to the PES topology. Moreover, if the quantum mechanical probability density for $\mathrm{H}$ shows two peaks because of the tunnelling process, the concept of vibrationally averaged structure for this atom loses its meaning.

In order to explore these effects for the protonated water 
clusters, we collected 1D and 2D probability density functions for the interatomic distances in the clusters. This is formally equivalent to computing the two integrals,

$$
\rho(\mu)=\int d \mathbf{R} \delta\left(\mu-\left|\mathbf{r}_{i}-\mathbf{r}_{j}\right|\right) \psi_{0}(\mathbf{R})
$$

and

$$
\Gamma(\mu, \nu)=\int d \mathbf{R} \delta\left(\mu-\left|\mathbf{r}_{i}-\mathbf{r}_{j}\right|\right) \delta\left(\nu-\left|\mathbf{r}_{l}-\mathbf{r}_{m}\right|\right) \psi_{0}(\mathbf{R}),
$$

where $\psi_{0}$ is the ground state wave function and $\mathbf{r}_{i}$ is the vector representing the position of the $i$ th atom. It is important to notice that, differently from the usual quantum expectation value, these values are defined using $\psi_{0}$ and not $\psi_{0}^{2}$. Although this hinders the possibility of interpreting $\rho$ and $\Gamma$ as true probability density functions, they still represent a useful tool to understand the importance of the quantum effects on the system behavior. ${ }^{46}$ However, instead of collecting simple interatomic distance density functions, one could cleverly select one or two internal coordinates so that the relative motion of atoms, or groups of atoms would be decoupled. If this were possible, $\rho$ and $\Gamma$ could be easily transformed into true probability density by simply squaring them. To illustrate this idea, we mention the concept of $\mathrm{H}$-density ${ }^{47}$ that was developed to visualize the quantum effects of the $\mathrm{H}$ atoms belonging to a water molecule solvating a biomolecule. Here, the sampled $\psi_{0}$ is collected for every $\mathrm{H}$ atom in a bin grid as to form a 4D histogram, and disregarding the position of all the other atoms. The value of the resulting histograms are successively squared to represent an approximation to the correct density of the hydrogen atoms. It is worth mentioning that exact and quasiexact approaches are also available to obtain informations on the probability density of $\mathrm{H}$ atoms. These range from the forward walking scheme,${ }^{48}$ to squaring the walker weights, ${ }^{46}$ to collecting a large set of replica and weighting them proportionally to the number of neighbor walkers within a defined distance in the configurational space. ${ }^{49}$ However, for the analysis of the effects that a quantum treatment has on the structural features of these systems we found the usage of $\rho$ and $\Gamma$ [Eqs. (9) and (10)] to convey sufficient informations and to make us able to distinguish between different local environments experienced by the protons.

As was mentioned previously, the study of the 1D and 2D distributions was also supplemented with the visual inspection of a subset of walkers during the production runs. This enables the direct observation of the conformational changes that take place, and was useful to understand that the oxygen atoms never exchanged the relative positions even during long runs. Although this finding was not unexpected due to the large oxygen mass and the high barrier that such a process must overcome, it allows us to use the oxygen "backbone" of the clusters as a reference to describe the hydrogen motion.

In Fig. 6, we show the 1D probability density distribution [Eq. (9)] of the oxygen-oxygen and oxygen-hydrogen distances for $\mathrm{H}_{5} \mathrm{O}_{2}^{+}$. For the $\mathrm{O}-\mathrm{H}$ distances, the distribution shows two well separated peaks which are due to the two

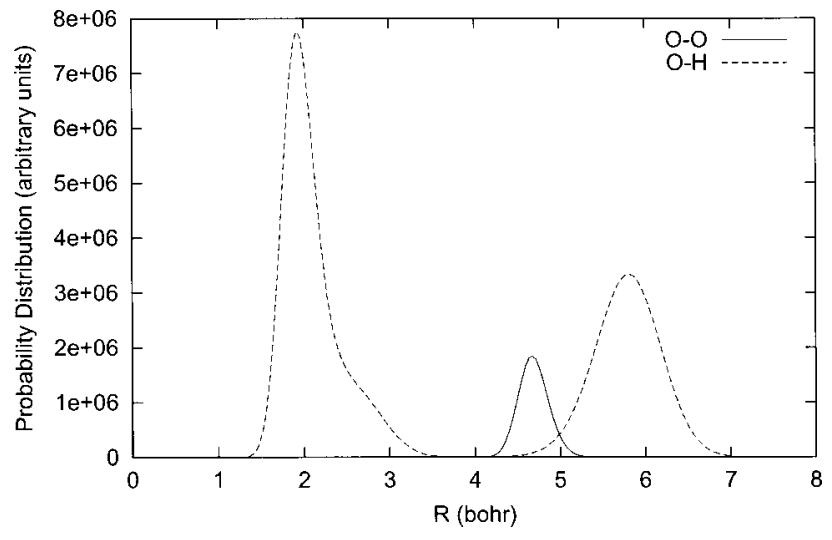

FIG. 6. Unnormalized DMC distribution function of the $\mathrm{OH}$ (dashed line) and $\mathrm{OO}$ (solid line) distances for $\mathrm{H}_{5} \mathrm{O}_{2}^{+}$. Distances in bohr.

distinct sets of hydrogen atoms that an $\mathrm{O}$ sees, namely the ones bonded to itself and the ones bonded to the other $\mathrm{O}$. Moreover, the peak located at short $\mathrm{O}-\mathrm{H}$ distance shows a well defined shoulder that can be attributed to the $\mathrm{H}$ atom lying midway between the oxygen atoms. At the equilibrium geometry, this $\mathrm{H}$ atom has a slightly longer bond distance from the $\mathrm{O}$ atoms than the other four hydrogens. As expected, the distribution of oxygen-hydrogen distances is fairly broad, indicating the importance of the relative quantum mechanical vibrational motion of the $\mathrm{H}$ atoms. Notwithstanding the larger oxygen mass, also the $\mathrm{O}-\mathrm{O}$ distance distribution clearly shows the effect due to the quantum zero point energy on the molecular structure extending from 4.3 to 5.3 bohr. Similar results were also found for the larger systems, but we did not include the relevant figures since these are quite cluttered and, especially for the largest clusters, they convey very small additional informations. Our results for the quantum distribution of these systems are consistent with previous DMC simulations on pure water clusters $^{50}$ and reinforce the idea that the quantum contribution to the motion of the first row atoms can be important in describing systems containing hydrogen bonds. ${ }^{51}$

To extract more information about the structural effect of the quantum motion, we also computed the $2 \mathrm{D}$ distribution obtained by using the two internal coordinates $Z=r\left(\mathrm{O}_{i} \mathrm{H}\right)$ $-r\left(\mathrm{O}_{j} \mathrm{H}\right)$ and $R=r\left(\mathrm{O}_{i} \mathrm{O}_{j}\right)$ for any $\mathrm{O}_{i} \mathrm{O}_{j}$ pair and any $\mathrm{H}$ atom in the system. Here, $r\left(\mathrm{O}_{i} \mathrm{H}\right)$ indicates the distance between the $i$ th $\mathrm{O}$ atom and a $\mathrm{H}$ atom, and $r\left(\mathrm{O}_{i} \mathrm{O}_{j}\right)$ indicate the distance between the $i$ th and $j$ th $\mathrm{O}$ atoms. This approach excludes any possible bias due to an incorrect instantaneous choice of the two reference $\mathrm{O}$ atoms.

The distribution obtained by simulating the Zundel cation is shown in Fig. 7. The two large and symmetric peaks centred around $R(\mathrm{OO})=4.70 \mathrm{bohr}$ and $Z=-4.4 \mathrm{bohr}$ represent the two pairs of $\mathrm{H}$ atoms lying externally to the $\mathrm{O}-\mathrm{O}$ framework and not involved in the bonding. The peak located at $Z=0$, instead, represents the central hydrogen equally shared between the two water molecules. Its distribution appears to be wider than the other two peaks, and for $R \geqslant 5 \mathrm{bohr}$, the split tail clearly indicates that $\mathrm{H}$ no longer experiences a single well potential but rather a double well one. Despite highlighting the features of the quantum 


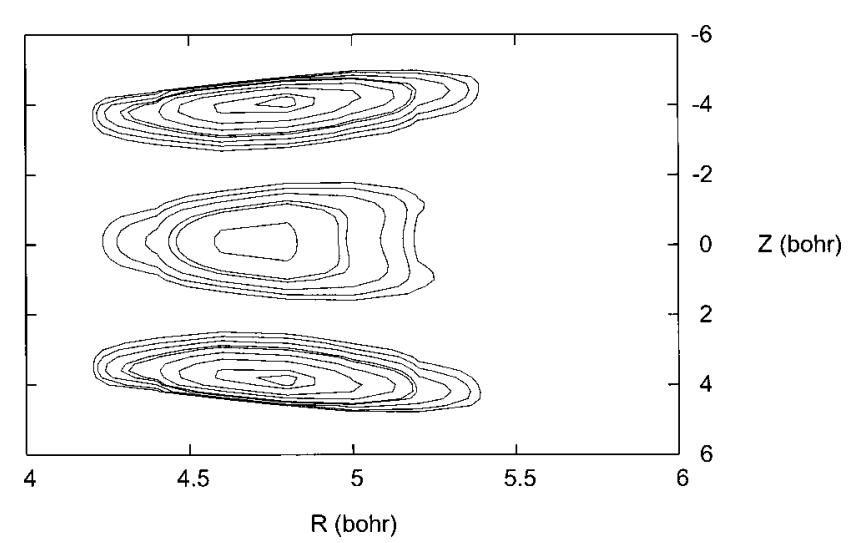

FIG. 7. Contour plot of the $2 \mathrm{D}$ distribution function for $\mathrm{H}_{5} \mathrm{O}_{2}^{+}$obtained using the internal coordinates $Z=r\left(\mathrm{O}_{i} \mathrm{H}\right)-r\left(\mathrm{O}_{j} \mathrm{H}\right)$ and $R=r\left(\mathrm{O}_{i} \mathrm{O}_{j}\right)$. The contours are drawn at $0.01,0.02,0.04,0.08,0.1,0.2,0.4,0.8$ times the maximum peak height. Distances in bohr.

$\mathrm{H}$-density for the Zundel cation $\mathrm{H}_{5} \mathrm{O}_{2}^{+}$, using $Z$ and $R$ as variables to define $\Gamma$ produces highly cluttered pictures for the larger clusters that are difficult to interpret. For this reason, we also define a new set of coordinates using $R$ $=r\left(\mathrm{O}_{i} \mathrm{H}\right)+r\left(\mathrm{O}_{j} \mathrm{H}\right)$ instead of $R=r\left(\mathrm{O}_{i} \mathrm{O}_{j}\right)$. This choice is reminiscent of the elliptical coordinates used in the quantum treatment of $\mathrm{H}_{2}$ and $\mathrm{H}_{2}^{+}$and has the advantage of relating the $\mathrm{H}$-density peak with the $\mathrm{H}$ atoms external to the $\mathrm{O}_{i}-\mathrm{H}-\mathrm{O}_{j}$ bond region at larger $R$. This makes the interpretation of the plotted density easier. Figure 8 shows the appearance of $\Gamma$ when the latter choice of coordinates is used. As clearly seen, the two peaks at $Z=-4$ and 4 are now well separated by the central peak describing the shared $\mathrm{H}$, whose maximum is located roughly at $R=4.8 \mathrm{bohr}$. Moreover, this one also displays the split tail indicating the presence of a double well potential, so we consider it appropriate and use only this last coordinate system to extract the largest amount of information from the sampled $\psi_{0}$.

As for $\left(\mathrm{H}_{2} \mathrm{O}\right)_{3} \mathrm{H}^{+}$, Fig. 9 partially shows one of the two equivalent $2 \mathrm{D}$ distributions including the central oxygen and one of the two $\mathrm{H}$ involved in hydrogen bonding with the external water molecules. Differently from the $\left(\mathrm{H}_{2} \mathrm{O}\right)_{2} \mathrm{H}^{+}$ cation, the peak around $R=5.3 \mathrm{bohr}$ is asymmetrical and its

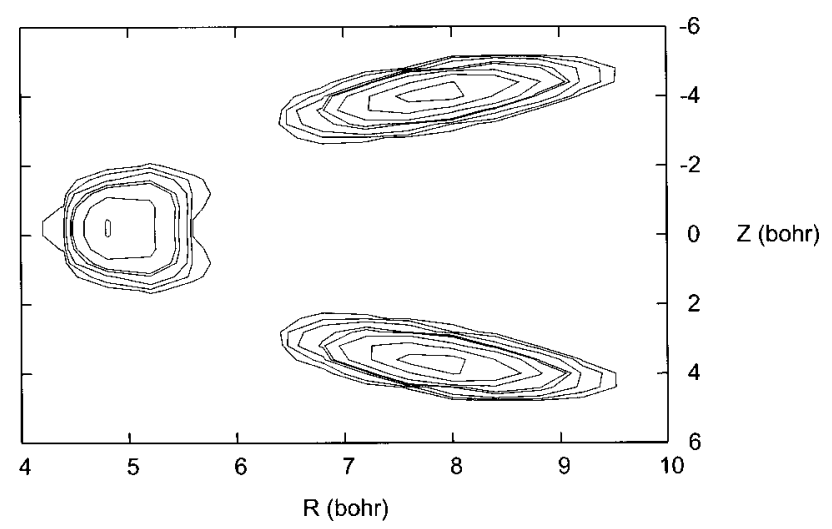

FIG. 8. Contour plot of the 2D distribution function for $\mathrm{H}_{5} \mathrm{O}_{2}^{+}$obtained using the internal coordinates $\mathrm{Z}=r\left(\mathrm{O}_{i} \mathrm{H}\right)-r\left(\mathrm{O}_{j} \mathrm{H}\right)$ and $R=r\left(\mathrm{O}_{i} \mathrm{H}\right)$ $+r\left(\mathrm{O}_{j} \mathrm{H}\right)$. The contours are drawn at $0.01,0.02,0.04,0.08,0.1,0.2,0.4,0.8$ times the maximum peak height. Distances in bohr.

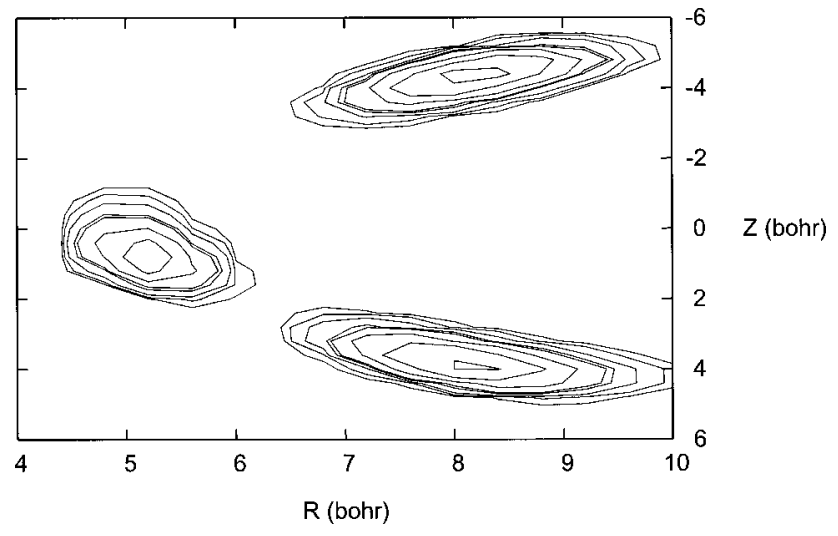

FIG. 9. Contour plot of the 2D distribution function for $\mathrm{H}_{7} \mathrm{O}_{3}^{+}$obtained using the internal coordinates $\mathrm{Z}=r\left(\mathrm{O}_{i} \mathrm{H}\right)-r\left(\mathrm{O}_{j} \mathrm{H}\right)$ and $R=r\left(\mathrm{O}_{i} \mathrm{H}\right)$ $+r\left(\mathrm{O}_{j} \mathrm{H}\right)$. Only the part of the distribution close to the hydrogen bond region between the central and one external $\mathrm{O}$ atoms is shown. The contours are drawn at $0.01,0.02,0.04,0.08,0.1,0.2,0.4,0.8$ times the maximum peak height. Distances in bohr.

maximum is located in the positive region of $Z$. Although this indicates that the $\mathrm{H}$ atom preferentially resides on the central $\mathrm{O}$ atom, the width of the peak strongly suggests that the bond region closer to the external $\mathrm{O}$ is visited by the hydrogen due to the quantum motion. Interestingly, the location of the peak maximum along $R$ is 0.4 bohr larger than in the Zundel cation case. In the last one, the smaller value of $R$ for the maximum probably indicates the shorter $\mathrm{O}-\mathrm{O}$ distance due to the strong $\mathrm{H}$ bond bridging the two oxygens in the system, which is relaxed upon the addition of a second water to the hydronium cation. This is consistent with the location of the first peak of the $\mathrm{O}-\mathrm{O}$ pair distribution function for both systems. Whereas the Zundel cation shows a maximum around 4.7 bohr (see Fig. 6), we found the $\mathrm{O}-\mathrm{O}$ distribution for the trimer to locate it at $4.9 \mathrm{bohr}$.

Moving on to discuss the two tetramers, Fig. 10 shows the probability distribution for the $\mathrm{H}$ atoms for the $\mathrm{O}$ pair of the two central water molecules in the linear $\left(\mathrm{H}_{2} \mathrm{O}\right)_{4} \mathrm{H}^{+}$. The broad peak centred at $Z=0$ bohr clearly resembles the one

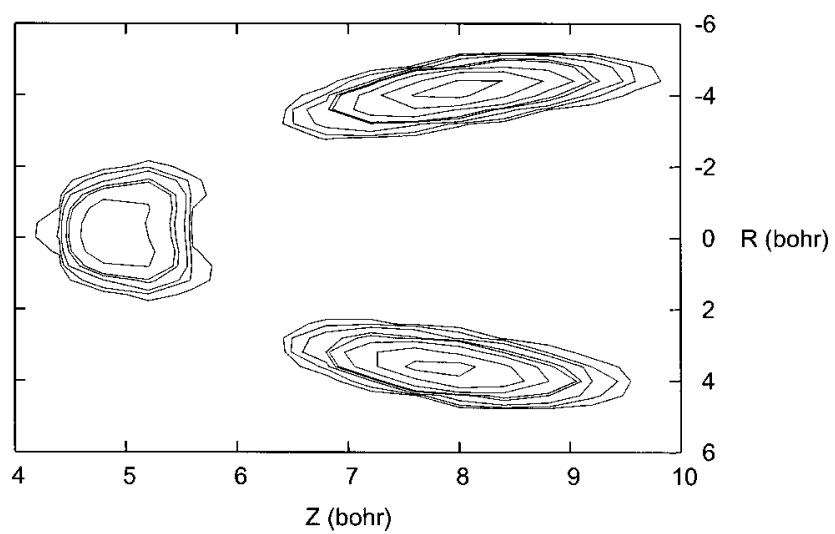

FIG. 10. Contour plot of the 2D distribution function for the linear $\mathrm{H}_{9} \mathrm{O}_{4}^{+}$ obtained using the internal coordinates $Z=r\left(\mathrm{O}_{i} \mathrm{H}\right)-r\left(\mathrm{O}_{j} \mathrm{H}\right)$ and $R$ $=r\left(\mathrm{O}_{i} \mathrm{H}\right)+r\left(\mathrm{O}_{j} \mathrm{H}\right)$. Only the part of the distribution close to the hydrogen bond region between the two central $\mathrm{O}$ atoms is shown. The contours are drawn at $0.01,0.02,0.04,0.08,0.1,0.2,0.4,0.8$ times the maximum peak height. Distances in bohr. 


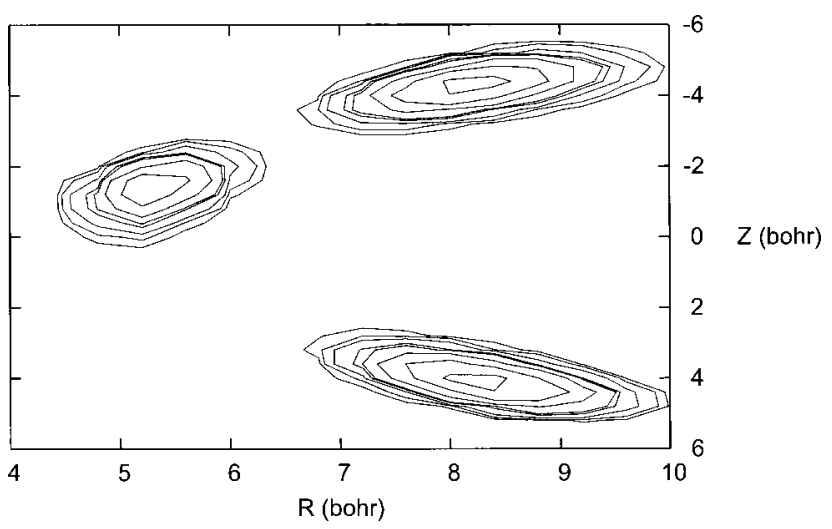

FIG. 11. Contour plot of the 2D distribution function for the branched $\mathrm{H}_{9} \mathrm{O}_{4}^{+}$obtained using the internal coordinates $\mathrm{Z}=r\left(\mathrm{O}_{i} \mathrm{H}\right)-r\left(\mathrm{O}_{j} \mathrm{H}\right)$ and $R$ $=r\left(\mathrm{O}_{i} \mathrm{H}\right)+r\left(\mathrm{O}_{j} \mathrm{H}\right)$. Only the part of the distribution close to the hydrogen bond region between the central and one external $\mathrm{O}$ atoms is shown. The contours are drawn at $0.01,0.02,0.04,0.08,0.1,0.2,0.4,0.8$ times the maximum peak height. Distances in bohr.

observed for the Zundel cation, therefore indicating the presence of a $\mathrm{H}$ between the two oxygens equally shared by means of a strong hydrogen bond. Also in this case, the split tail indicates that the proton visits regions of the PES where a double well is present. The other hydrogens involved in hydrogen bonding between the central $\left(\mathrm{H}_{2} \mathrm{O}\right)_{2} \mathrm{H}^{+}$moiety and the external water molecules show a distribution similar to the $\left(\mathrm{H}_{2} \mathrm{O}\right)_{3} \mathrm{H}^{+}$case, although relatively less diffuse. As far as the branched tetramer is concerned, the distribution function for one of the three equivalent $\mathrm{O}-\mathrm{O}$ pairs containing the central oxygen is shown in Fig. 11. Not unexpectedly, it closely resembles the trimer density. This is due to the fact that in both systems the charge bearing entity can be considered the hydronium ion $\mathrm{H}_{3} \mathrm{O}^{+}$. However, the distribution of the hydrogen bonded $\mathrm{H}$ is less diffuse in the branched $\left(\mathrm{H}_{2} \mathrm{O}\right)_{4} \mathrm{H}^{+}$than in $\left(\mathrm{H}_{2} \mathrm{O}\right)_{3} \mathrm{H}^{+}$, indicating a more compact $\mathrm{H}_{3} \mathrm{O}^{+}$.

Distributions similar to the ones for the trimer and the tetramer are also found for the two isomers of the pentamer, so they are not shown. This outcome is explained by the close similarity between the minimum energy structure of the trimer and linear pentamer, and the branched $\left(\mathrm{H}_{2} \mathrm{O}\right)_{4} \mathrm{H}^{+}$and $\left(\mathrm{H}_{2} \mathrm{O}\right)_{5} \mathrm{H}^{+}$. However, the H-density of the central water molecule in the linear isomer pentamer is slightly broader in the negative $Z$ region than the trimer one, indicating that the inner turning point of the $\mathrm{H}$ motion is located closer to the central $\mathrm{O}$ atom in the pentamer than in the trimer case. Conversely, the branched isomer distribution for the central hydronium is almost perfectly superimposable with the one for the branched tetramer, indicating that the fifth water molecule located in the "second solvation shell" does not play an important role in defining the quantum motion of the $\mathrm{H}_{3} \mathrm{O}^{+}$moiety. A close similarity is also seen by comparing the distribution for the $\mathrm{H}$ atoms involved in hydrogen bonds with the "second solvation shell" water molecules in the two pentamers, therefore indicating that the $\mathrm{H}$ atoms are experiencing similar environments.

\section{Ring opening}

As mentioned before, following the simulation procedure previously outlined, we found that during the long production runs, the cyclic isomers opened the ring, giving either a linear or a branched chain isomers. Moreover, at the end of the DMC simulations we found that the population of cyclic isomers completely disappeared from the walker ensemble. In order to test if these findings were only an artifact of the parameters of the simulation method, we carried out more simulations modifying either the number of walkers or the time step. Similar results were obtained in all the cases, therefore indicating the ring opening as a solid feature of the OSS3 potential and not a shortcoming of the simulations. At this point, it is important to remember that, given the chance to explore all the configuration space, DMC samples the ground state wave function for a chosen Hamiltonian. Since we always started a simulation by generating a set of walkers using the local equilibrium geometry of given isomer, the ring opening process is directly related to the flowing of the probability density function through the energy barrier. In turn, this indicates that, while existing as well defined geometrical isomers due the topology of the potential energy surface, the addition of the vibrational quantum effects to the cyclic systems allows them to cross the energy barrier that separates the basins of attraction for the two isomeric complexes.

To quantitatively characterize this process, we localized the transition state (TS) geometry for the ring opening by minimizing the square modulus of the PES gradient. We computed the PES gradient $\nabla V(\mathbf{R})$ by means of a finite difference procedure based on the third order formula $\partial f(x) / \partial x=[f(x+h)-f(x-h)] / 2 h$. The $[\nabla V(\mathbf{R})]^{2}$ minimization process was carried out by employing, once more, the Powell's algorithm. Although easier to program than other second order methods used to locate TS, minimizing the square modulus of the gradient requires a good starting point well inside the basin of attraction of the TS. In order to obtain a good guess for the TS, we exploited the fact that, monitoring the walkers during the simulations, it was noticed that the most noticeable change was the increase of the distance between two separating oxygens connected by a $\mathrm{H}$ bond. So, a constrained optimization was carried out for many different values of this distance to compute a "reaction profile" along it, and the one obtained for the tetramer ring is shown in Fig. 12. As it is clearly seen, it is possible to identify two minima and a maximum of the potential energy along this cut. It is also worth noticing that the two wells for the closed and open isomers are quite different in terms of their curvature with respect to changes in the $\mathrm{O}-\mathrm{O}$ distance. This feature is related to the fact that, once passed the TS, increasing the $\mathrm{O}-\mathrm{O}$ distance has an effect almost equivalent to changing the torsional angle between the two oxygen atoms involved in the process. Since the potential in that zone of configuration space appears to be shallow with respect to varying the torsional angle, this produces the less curved second well shown in Fig. 12. Largely similar results are obtained for the all the cyclic pentamers.

To refine the geometry and energy of the TS, we started from the structure of the system close to the maximum pro- 


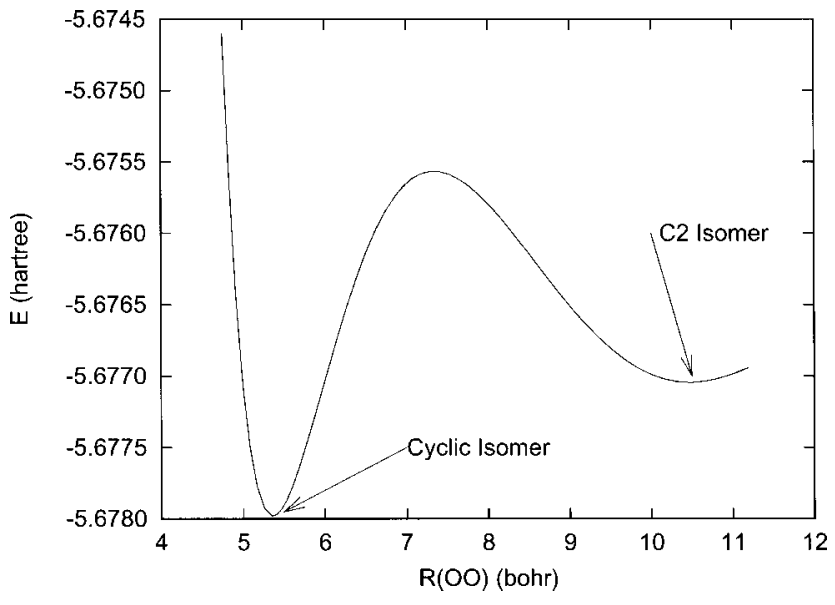

FIG. 12. Approximate reaction profile obtained by constrained optimization on the OSS3 PES. The reaction coordinate is chosen to be the distance between the two separating $\mathrm{O}$ atoms. Quantities in a.u.

duced by the constrained optimization procedure, obtaining a fast convergency to a minimum of the square modulus of the gradient. Also, the local minimum in the open isomer generated by the constrained minimization was fully optimized to compare with the energy minima shown in Table I, and the complete set of results are shown in Table V.

Comparing the two sets of energy results for the minima in Table I and Table V, it appears that the open isomer generated by the ring breaking of $4^{(2)}, 5^{(1)}$, and $5^{(5)}$ differ from any of previously optimized ones. Indeed, a visual inspection proved the pentamers to differ only by the relative orientation of a terminal water molecule which is easily changed during the DMC simulation. Conversely, the tetramer opening gives rise to a linear gauche isomer while the most stable one is anti. However, these two isomers are readily converted during the DMC simulation.

As for the energy barriers $\Delta E^{\ddagger}$ shown in Table $\mathrm{V}$, we define the direct process as the one involving the ring opening, and $\Delta E_{\text {direct }}^{\ddagger}$ as the energy difference between the ring isomer and the transition state. Conversely, inverse indicates the ring closing process, and $\Delta E_{\text {inverse }}^{*}$ is the energy difference between the transition state and the open isomer. As one could notice, both the direct and inverse processes have low barrier heights, the lowest direct one being the one related to the opening of the $5^{(3)}$ cyclic pentamer, namely 1.1 mhartree (roughly $0.7 \mathrm{kcal} / \mathrm{mol}$ ). These results explain why the DMC simulations were able to produce the open isomers. Clearly, for such a low barrier height, even a small amount of quan- tum kinetic energy injected in the relative motion of two oxygens is enough to surmount the barrier on going from the cyclic isomer to the open one.

Interestingly, all but the $5^{(3)}$ isomer opened into a linear or branched complex having a higher minimum potential energy. Recalling that the end of each DMC simulation we found only linear or branched complexes instead of a mixture containing also the ring as one would expect on the basis of the relative adiabatic stability, we consider this finding to be a counterintuitive result that deserves a careful explanation. In order to do so, we make use of the harmonic approximation and compute the ZPE(HA) for all the energy minima (see Table I). Comparing the ZPE(HA) for the various systems, it clearly appears that the cyclic isomers possess a larger vibrational energy than the open ones. This is probably due to the fact that cyclic systems are usually more rigid and therefore vibrate at higher frequencies. Although this difference could easily explain why the linear tetramer is more stable than the cyclic one and why $5^{(4)}$ opens up giving rise to the linear pentamer, the order of stability for the other protonated clusters, although compressed, remains unchanged. In order to account for the DMC stability results, one must remember that for the branched and linear pentamers, the anharmonicity of the potential accounts for a decrease in the ZPE of roughly 3.3 (2.1) and 5.6 (3.5) mhartree $(\mathrm{kcal} / \mathrm{mol})$, respectively. We expect this decrease to be partially due to the larger conformational freedom acquired by allowing for a wider relative torsional motion of the molecular skeleton upon breaking of the ring. In turn, this may have the effect of reducing the overall curvature of the nuclear wave function, and hence the mean value of the kinetic energy, therefore stabilizing the branched and linear isomers.

Employing a completely different point of view, one could ask if there is any relationship between the structure of the cyclic complex and the bond that is going to break during the opening process. In order to answer this question, we start by noticing that all hydrogen bonds broken during the conversion were connecting a $\mathrm{H}_{3} \mathrm{O}^{+}$"first shell" water molecule with a double acceptor $\mathrm{H}_{2} \mathrm{O}$. We were able to notice this outcome by looking at the connectivity between $\mathrm{O}$ atoms which is uniquely defined by their labels. Moreover, by observing the optimized isomer geometries it emerges that this fact would be consistent with the idea of leaving the $\mathrm{H}_{3} \mathrm{O}^{+}$ moiety to be as much solvated as possible during the ring opening. However, due to the chance that the excess proton could migrate from an oxygen to a neighbor one during the opening process, it is possible that a bond breaking different

TABLE V. OSS3 energetics for the ring opening and closing of the cyclic $\left(\mathrm{H}_{2} \mathrm{O}\right)_{n} \mathrm{H}^{+}$isomer. The column of the $V_{\min }$ (open) energy values also contains the label indicating the open isomer obtained at the end of the ring opening. For a detailed description of the processes see main text. Quantities in a.u. apart from the barrier heights in square brackets that are give in $\mathrm{kcal} / \mathrm{mol}$.

\begin{tabular}{lccccc}
\hline \hline$n$ & $V_{\min }($ ring $)$ & $V_{\text {saddle }}$ & $V_{\min }($ open $)$ & $\Delta E_{\text {direct }}^{\ddagger}$ & $\Delta E_{\text {inverse }}^{\ddagger}$ \\
\hline $4^{(2)}$ & -5.677980 & -5.675566 & $-5.677044\left(4^{(3)}\right)$ & $0.002414[1.51]$ & $0.001478[0.93]$ \\
$5^{(1)}$ & -7.052958 & -7.049763 & $-7.050669\left(5^{(2)}\right)$ & $0.003195[2.00]$ & $0.000906[0.57]$ \\
$5^{(3)}$ & -7.0499961 & -7.048882 & $-7.051081\left(5^{(2)}\right)$ & $0.001079[0.67]$ & $0.002199[1.38]$ \\
$5^{(4)}$ & -7.049823 & -7.045501 & $-7.048409\left(5^{(6)}\right)$ & $0.004322[2.71]$ & $0.002908[1.82]$ \\
$5^{(5)}$ & -7.049226 & -7.045496 & $-7.048054\left(5^{(6)}\right)$ & $0.003730[2.34]$ & $0.002558[1.60]$ \\
\hline \hline
\end{tabular}


from the one obtained in the simulation may create a structure presenting a similar solvation pattern or even an identical open isomer. So, we suspect that another factor is also playing a role, namely the height of the barriers that must be overcome to produce the open isomer. To help discriminating between the relative importance of the two effects, a further energetic analysis has been made for all the cyclic isomers. In this analysis, we carried out a partial optimization of all the cyclic systems constraining, one by one, the "unique by symmetry" $\mathrm{O}-\mathrm{O}$ ring distances to produce PES scans similar to the ones used in the TS calculations. In all the cases but one deriving from $5^{(3)}$, we notice that none of the two oxygen atoms whose distance was constrained during the scan belonged to the double acceptor water. Also, the value of potential energy along the scanned internal coordinate rised well above $(7-13 \mathrm{kcal} / \mathrm{mol})$ the barrier height listed in Table $\mathrm{V}$ for the specific isomer studied. Notwithstanding the fact that the $\mathrm{O}-\mathrm{O}$ distance may be only an approximation to the exact reaction coordinate of the opening process in the reactant region, this finding seems to indicate that, to open the ring, the DMC simulation took the path passing through the barrier having the lowest height while, at the same time, keeping the $\mathrm{H}_{3} \mathrm{O}^{+}$as much solvated as possible. This is also consistent with the fact that the DMC algorithm kills the walkers visiting regions where the local potential is higher that the average over the ensemble. We also found that, during the scan, all the systems underwent an internal conversion by means of which the double acceptor water molecule became donor-acceptor by swapping one of the H-bond with neighbor molecule. This finding suggests that other pathways are indeed available for the ring opening. However, these seem to require surmounting barriers higher than the ones presented in Table V, so that they are less likely to be followed in a DMC simulation. Although the study of these additional pathways is interesting on its own, we consider it to be outside the purpose of this work and we plan to tackle it in a future work.

As to $5^{(3)}$, this system has two possible pathways available for the ring opening involving the double acceptor water molecule, namely one producing the branched isomer $5^{(2)}$ found at the end of the simulation, and another one leading to the linear $5^{(6)}$ one. Knowing that the correct description is given by the $5^{(3)} \rightarrow 5^{(2)}$ process (i.e., the one that produces the most stable isomer), we recognize that this transformation is under the "thermodynamical" control mentioned before, i.e., the process evolves keeping the hydronium fully solvated. However, it must be pointed out that the difference between the equilibrium potential energy of $5^{(3)}$ and $5^{(6)}$ is already 0.5 mhartree $(0.3 \mathrm{kcal} / \mathrm{mol})$ larger than the barrier for the "simulated" $5^{(3)} \rightarrow 5^{(2)}$ ring opening. So, one should expect the barrier of the $5^{(3)} \rightarrow 5^{(6)}$ process to be, at least, 2.53.5 mhartree $(1.6-2.2 \mathrm{kcal} / \mathrm{mol})$. Indeed, optimizing the TS for the $5^{(3)} \rightarrow 5^{(6)}$ process we found a barrier height of 3.15 mhartree $(2 \mathrm{kcal} / \mathrm{mol})$. Once more, this suggests that DMC took the pathway with the lowest barrier to open the ring.

\section{DISCUSSION AND CONCLUSIONS}

In this work, a thorough study of the structure and energetics of the protonated water clusters as described by the
OSS3 potential was carried out. Although the quality of our results strongly relies on the accuracy of the OSS3 model, the accurate treatment of the total and binding energy and of the equilibrium structures generated by this analytic potential convinces us that the global physical picture is semiquantitatively described.

Introducing the exact treatment of the ground state vibrational motion using the DMC method, we are able to highlight the importance of the ZPE with respect to the energetics of the protonated complexes. More specifically, the vibrational motion induces changes of the order of $2-12$ mhartree $(1.2-7.5 \mathrm{kcal} / \mathrm{mol})$ in the formation energy and proton affinity of the clusters when compared with the similar adiabatic quantities. Also, we found the effect of anharmonicity to account for variations in the energetics strongly dependent on the specific system. These changes are found to span the range $1.5-5.5$ mhartree $(0.9-3.5 \mathrm{kcal} / \mathrm{mol})$. In turn, this indicates that, in order to compute energetic quantities with a total accuracy of roughly $1 \mathrm{kcal} / \mathrm{mol}$ (i.e., chemical accuracy) for the stable systems, one must take anharmonicity into account. Even more importantly, we found all the cyclic isomers of these complexes to open their ring upon addition of ZPE by means of the exact DMC method. This finding is related to the fact that DMC samples the exact ground state wave function $\Psi_{0}$ for a given Hamiltonian, and that the cyclic and open isomers are usually separated by low energy barriers spanning the $1-4.3$ mhartree $(0.6-2.7 \mathrm{kcal} /$ mol) range. However, we stress that this outcome is due to the interplay between the possibility of tunneling between PES minima and the dynamical stabilization generated in one of the potential energy wells by having a smaller curvature than the others. It can be also seen as an indication of the failure of the harmonic approximation in correctly introducing the quantum vibrational motion in these systems as recently highlighted in a quantum-thermal study of a biomolecule. ${ }^{52}$ In this work the inversion of the stability order was explained on the basis of an entropic stabilization of one of the conformers with respect to the others having a closely similar potential energy minimum but a smaller local curvature.

Let us now turn to discuss the relevance of our results for the many different fields related to the study of protonated species. As already mentioned, our findings strongly suggest that, in order to have accurate thermochemical quantities, the anharmonicity of the adiabatic energy surface must be taken into account, and this becomes more important the larger the cluster. In turn, this indicates that the standard $a b$ initio plus harmonic approximation will not suffice for this task, and an approach interfacing ab initio with some accurate treatment of the anharmonicity must be sought. Moreover, remembering that our results are obtained modelling the protonated water clusters at $0 \mathrm{~K}$, one should expect that the correct treatment of anharmonicity to describe the thermochemistry will become even more important at higher temperatures due to the thermal excitation involved.

The ring opening process evidenced during our simulations introduces another point of concern with respect to the standard modelling approach, namely the necessity of studying the energy barriers and the pathways connecting two dif- 
ferent isomers. This is necessary to predict if an isomer is stable or not with respect to the inclusion of the ZPE, and what is the preferred mechanism of ring opening. At this stage, it is important to point out that we use the term "stability" for one isomer in a slightly unusual way. Bearing in mind that every simulation was started from a local minimum of the OSS3 PES, we use "stability" to indicate the constant presence of a fraction of the walker population inside the basin of attraction of the starting stationary point (isomer) during all the duration of the DMC simulation. If more than one isomer is found at the end of the simulation, we consider this as indicating that the ground state of the system must be represented as a mixture of two or more coexisting isomeric species. Instead, one should consider the absence of walkers having the starting isomeric structure as merely representing the fact that the final species obtained dominates the population of the ground state, and that the basin of attraction of the starting isomer may become populated only as the result of a vibrational excitation. A practical procedure to estimate the energy of the excited state that would populate the basin of the starting isomer is given by killing walkers passing through the barrier. This is used as a way to stabilize the starting isomer and associate an energy value to it. However, given the small energy barrier separating the various stationary point on the OSS3 surface, we do not feel confident in using the technique. Due to the chance that the excited state populating the particular basin can be spatially extended and the location of its nodal surface difficult to predict, we consider necessary to use a more accurate excited state procedure to estimate its energy. Apart from this, at low temperature [e.g., $170 \mathrm{~K}$ (Ref. 7)] the amount of thermal energy available to excite a complex in a higher vibrational state is small. So, the vibrational ground state extracted by the DMC simulations is expected to dominate the population of isomers, and hence the system properties. In this sense, one should expect that, if the isomeric structure used to start the DMC simulation depopulates during the run it should also have a small thermal equilibrium population at low temperature, and it should convert into the ground state structure (hence to be "unstable" with respect the last one) while the system reaches thermal equilibrium. The latter would happen, obviously, only in the case the "unstable" isomer is formed as the first step of the cluster synthesis.

In comparing with experiments, our results have importance in the context of the direct thermochemical measures ${ }^{10,11}$ of the hydratation enthalpy and free energy for charged ions like $\mathrm{NH}_{4}^{+}$and $\mathrm{H}_{3} \mathrm{O}^{+}$. As to our results, the grow energy $E_{\text {grow }}$ is the $0 \mathrm{~K}$ equivalent of both enthalpy and free energy changes. However, not accounting for the increase of the available states due to thermal excitation (i.e., entropy), it can be compared only with $\Delta H^{0}$. Here, we do this with the most recent results available. ${ }^{11}$ Our $E_{\text {grow }}$ results ( $\mathrm{kcal} / \mathrm{mol})$ for the process of adding a single water molecule $((n-1) \rightarrow n)$ are: $31.4(1 \rightarrow 2), 21.2 \quad(2 \rightarrow 3), 16.8 \quad(3$ $\rightarrow 4), 11.5(4 \rightarrow 5)$. Here, we computed the energy difference only between the most stable tetramer and pentamer assuming chemical equilibrium in the gas phase. The $\Delta H^{0}$ experimental results $(\mathrm{kcal} / \mathrm{mol})$ for the same processes read: $31.8(1 \rightarrow 2), 19.0(2 \rightarrow 3), 17.6(3 \rightarrow 4), 11.5(4 \rightarrow 5)$. Not- withstanding the lack of thermal excitation in our results, it is possible to see that they are in reasonable agreement with the experimental results. Apart from the $(2 \rightarrow 3)$ process where experiments and theory differ by $2.2 \mathrm{kcal} / \mathrm{mol}$, the differences are well below the standard chemical accuracy. Nevertheless, an estimate of the thermal and mechanical contributions to the enthalpy differences (i.e., the difference between our $E_{\text {grow }}$ and $\Delta H^{0}$ ) can be easily obtained by using the harmonic approximation, assuming classical behavior for translation and rotation, and considering the system as a perfect gas. Using the frequencies computed by employing the OSS3 potential, the thermal correction to our anharmonic energy differences at the standard state are: $(1 \rightarrow 2) 1.4 \mathrm{kcal} /$ $\mathrm{mol},(2 \rightarrow 3) 0.6 \mathrm{kcal} / \mathrm{mol},(3 \rightarrow 4) 0.3 \mathrm{kcal} / \mathrm{mol}$, and $(4$ $\rightarrow 5) 0.5 \mathrm{kcal} / \mathrm{mol}$. These are all positive, therefore increasing slightly our estimate for the reaction enthalpies. The corrections reduce somehow the agreement between experiments and theory for the two process $(1 \rightarrow 2)$ and $(2 \rightarrow 3)$. However, it must be pointed out that anharmonicity could play an important role in the description of the thermal excitation of the inter and intramolecular vibrations, so that it would be interesting to have a finite temperature path integral Monte Carlo calculation to fully understand the quality of the OSS3 potential in reproducing the energetics of the hydratation process. As far as the $(2 \rightarrow 3)$ process is concerned, the relatively large discrepancy between the computed and experimental results may be explained by the superposition of two simultaneous factors, namely, the experimental error (roughly $1 \mathrm{kcal} / \mathrm{mol}$, Ref. 11 ), and the fact that the OSS3 potential, parametrized on the basis of $a b$ initio results for $\mathrm{H}_{5} \mathrm{O}_{2}^{+}$, slightly underestimate the formation energy of the Zundel cation when compared with the MP2 results (see Table III).

Our results also shed a different light on the experimental results obtained by recording infrared spectra of protonated water clusters presented in Ref. 7. There, the authors were discussing the possibility of finding a symmetrically shared proton in the $\left(\mathrm{H}_{2} \mathrm{O}\right)_{n} \mathrm{H}^{+}(n=5-8)$ by looking at some spectroscopic features at $170 \pm 20 \mathrm{~K}$. On the basis of comparisons between the experiments and the harmonic DFT vibrational spectra, they eventually proposed two cyclic complexes, one heptamer and one octamer, as candidates exhibiting a highly symmetric proton environment. Before discussing the relevance of our results with respect to the experiments, it is important to point out that the level of theory used to interprete the spectra, namely, B3LYP/6-31G*, might be considered insufficient to correctly describe the relative energetics, and hence the spectroscopic features of these systems. As an example, we mention that for the pentamers, the energy ordering between the four membered ring and the branched isomer obtained in Ref. 7 is reversed with respect to the one obtained by Christie and Jordan using B3LYP/aug-cc-pVTZ. ${ }^{16}$ Nevertheless, much of the supporting arguments for the two cyclic isomers were based on the relative stability of the single-ring isomers in the heptamer and octamer family, a concept strongly connected with the validity of the local structural hypothesis and harmonic approximation. On the basis of the results obtained by means of DFT calculations, ${ }^{7}$ it was also pointed out that one should 
expect the linear and branched isomers to become thermodynamically more stable than the ring ones upon increasing of the temperature. Indeed, both Christie and Jordan, and Ciobanu et al., showed that the protonated clusters may loose any ring structure already around a temperature of 150 $\mathrm{K}$, therefore indicating a fundamental fragility of the cubic and cyclic structures. We show, instead, that this fragility could be seen already at $0 \mathrm{~K}$ in the smaller systems, and that it is related only to the fact that the local structure hypothesis and the harmonic oscillator model are not valid approximation to describe this systems. So, as previously explained, the thermal excitations may not be necessary or play only a minor role in defining the geometry of a clusters at low temperature. $^{7}$

We hope that our findings will give rise to a more clear interpretation of the past and future experimental results on these interesting systems. Also, they could suggest thorough experiments to probe the proton dynamics in larger clusters.

\section{ACKNOWLEDGMENTS}

M.M. acknowledges an EPSRC Advanced Research Fellowship (GR/R77803/01) and Richard A. Christie for making available some unpublished results. M.M. also acknowledges Thomas F. Miller III for many useful discussions.

${ }^{1}$ A. A. Kornyshev, A. M. Kuznetsov, E. Spohr, and J. Ulstrup, J. Phys. Chem. B 107, 3351 (2003).

${ }^{2}$ I. Ivanov and M. L. Klein, J. Am. Chem. Soc. 124, 13380 (2002).

${ }^{3}$ R. Pomes and B. Roux, J. Phys. Chem. 100, 2519 (1996).

${ }^{4}$ H. S. Mei, M. E. Tuckerman, D. E. Sagnella, and M. L. Klein, J. Phys. Chem. B 102, 10446 (1998).

${ }^{5}$ S. Hammes-Schiffer, Acc. Chem. Res. 34, 273 (2001).

${ }^{6}$ A. R. Fersht, Structure and Mechanism in Protein Science: A Guide to Enzyme Catalysis and Protein Folding (Freeman, New York, 1999).

${ }^{7}$ J.-C. Jiang, Y.-S. Wang, H.-C. Chang, S. H. Lin, Y. T. Lee, G. NiednerSchatteburg, and H.-C. Chang, J. Am. Chem. Soc. 122, 1398 (2000).

${ }^{8}$ C.-C. Wu, C. Chaudhuri, J. C. Jiang, Y. T. Lee, and H.-C. Chang, J. Chin. Chem. Soc. (Taipei) 49, 769 (2002).

${ }^{9}$ P. Kebarle, S. K. Searles, A. Zolla, J. Scarborough, and M. Arshadi, J. Am. Chem. Soc. 89, 6393 (1967).

${ }^{10}$ Y. K. Lau, S. Ikuta, and P. Kebarle, J. Am. Chem. Soc. 104, 1462 (1982).

${ }^{11}$ M. Meot-ner and C. V. Speller, J. Phys. Chem. 90, 6616 (1986).

${ }^{12}$ S. E. Rodriguez-Cruz, R. A. Jockusch, and E. R. Williams, J. Am. Chem. Soc. 120, 5842 (1998).

${ }^{13}$ S. J. Singer, S. McDonald, and L. Ojaäe, J. Chem. Phys. 112, 710 (2000).

${ }^{14}$ C. V. Ciobanu, L. Ojamäe, I. Shavitt, and S. J. Singer, J. Chem. Phys. 113, $5321(2000)$.
${ }^{15}$ J. K. Gregory and D. C. Clary, J. Phys. Chem. 100, 18014 (1996).

${ }^{16}$ R. A. Christie and K. D. Jordan, J. Phys. Chem. A 105, 7551 (2001).

${ }^{17}$ S. McDonald, L. Ojamäe, and S. J. Singer, J. Phys. Chem. A 102, 2824 (1998).

${ }^{18}$ R. A. Christie and K. D. Jordan, J. Phys. Chem. A 106, 8376 (2002).

${ }^{19}$ R. Car and M. Parinello, Phys. Rev. Lett. 55, 2471 (1985).

${ }^{20}$ L. Ojamäe, I. Shavitt, and S. J. Singer, Int. J. Quantum Chem. 29, 657 (1995)

${ }^{21}$ L. Ojamäe, I. Shavitt, and S. J. Singer, J. Chem. Phys. 109, 5547 (1998).

${ }^{22}$ G. Corongiu, R. Kelterbaum, and E. Kochanski, J. Phys. Chem. 99, 8038 (1995).

${ }^{23}$ D. W. Schwenke and H. Partridge, J. Chem. Phys. 113, 6592 (2000).

${ }^{24}$ M. P. Hodges, A. J. Stone, J. Chem. Phys. 110, 6766 (1999).

${ }^{25}$ S. L. Fornili, M. Migliore, and M. A. Palazzo, Chem. Phys. Lett. 125, 419 (1986).

${ }^{26}$ R. E. Kozack and P. C. Jordan, J. Chem. Phys. 96, 3131 (1992).

${ }^{27}$ F. H. Stillinger and C. W. David, J. Chem. Phys. 69, 1473 (1978); 73, 3384 (1980).

${ }^{28}$ F. H. Stillinger and T. A. Weber, Chem. Phys. Lett. 79, 259 (1981).

${ }^{29}$ A. Warshel and R. M. Weiss, J. Am. Chem. Soc. 102, 6218 (1980).

${ }^{30}$ D. E. Sagnella and M. E. Tuckerman, J. Chem. Phys. 108, 2073 (1998).

${ }^{31}$ U. W. Schmitt and G. A. Voth, J. Chem. Phys. 111, 9361 (1999).

${ }^{32}$ T. J. F. Day, A. V. Soudackov, M. Cûma, U. W. Schmitt, and G. A. Voth, J. Chem. Phys. 117, 5839 (2002).

${ }^{33}$ B. L. Hammond, W. A. Lester Jr., and P. J. Reynolds, Monte Carlo Methods in Ab Initio Quantum Chemistry, 1st ed. (World Scientific, Singapore, 1994).

${ }^{34}$ J. B. Anderson, J. Chem. Phys. 63, 1499 (1975).

${ }^{35}$ H. De Raedt and B. De Raedt, Phys. Rev. A 28, 3575 (1983).

${ }^{36}$ M. Mella, G. Morosi, and D. Bressanini, Phys. Rev. E 61, 2050 (2000).

${ }^{37}$ S. Chiesa, M. Mella, G. Morosi, and D. Bressanini, J. Chem. Phys. 119, 5601 (2003).

${ }^{38}$ H. A. Forbert and S. A. Chin, Int. J. Mod. Phys. B 15, 1752 (2001).

${ }^{39}$ J. H. Hetherington, Phys. Rev. A 30, 2713 (1984).

${ }^{40}$ Matthew P. Hodges and David J. Wales, Chem. Phys. Lett. 324, 279 (2000); http://wwwwales.ch.cam.ac.uk/wales/CCD/H3O+..H2ON/ gmin.html

${ }^{41}$ S. S. Xantheas, C. J. Burnham, and R. J. Harrison, J. Chem. Phys. 116, 1493 (2002)

${ }^{42}$ A. B. Finnila, M. A. Gomez, C. Sebenik, C. Stenson, and J. D. Doll, Chem. Phys. Lett. 219, 343 (1994).

${ }^{43}$ H. Flyvbjerg and H. G. Peterson, J. Chem. Phys. 91, 461 (1989).

${ }^{44} \mathrm{R}$. A. Christie (private communication). The values of the formation energy for the linear and branched tetramers computed using B3LYP/augcc-pVTZ are 0.1197 hartree and 0.1249 hartree, respectively.

${ }^{45}$ K. R. Glaesemann and L. E. Fried, J. Chem. Phys. 118, 1596 (2003).

${ }^{46}$ M. A. Suhm and R. O. Watts, Phys. Rep. 204, 293 (1991).

${ }^{47}$ D. C. Clary, D. M. Benoit, and T. Van Mourik, Acc. Chem. Res. 33, 441 (2000).

${ }^{48}$ M. H. Kalos, J. Comput. Phys. 2, 257 (1967).

${ }^{49}$ V. Buch, P. Sandler, and J. Sadlej, J. Phys. Chem. B 102, 8641 (1998).

${ }^{50}$ J. K. Gregory and D. C. Clary, J. Phys. Chem. 100, 18014 (1996).

${ }^{51}$ M. E. Tuckerman and D. Marx, Phys. Rev. Lett. 86, 4946 (2001).

${ }^{52}$ T. F. Miller III and D. C. Clary (unpublished). 